\title{
Discreteness of interior transmission eigenvalues revisited
}

\author{
Hoai-Minh Nguyen $^{1}$ • Quoc-Hung Nguyen ${ }^{1}$
}

Received: 13 December 2016/ Accepted: 20 February 2017 / Published online: 22 March 2017

(C) Springer-Verlag Berlin Heidelberg 2017

\begin{abstract}
This paper is devoted to the discreteness of the transmission eigenvalue problems. It is known that this problem is not self-adjoint and a priori estimates are non-standard and do not hold in general. Two approaches are used. The first one is based on the multiplier technique and the second one is based on the Fourier analysis. The key point of the analysis is to establish the compactness and the uniqueness for Cauchy problems under various conditions. Using these approaches, we are able to rediscover quite a few known discreteness results in the literature and obtain various new results for which only the information near the boundary are required and there might be no contrast of the coefficients on the boundary.
\end{abstract}

\section{Contents}

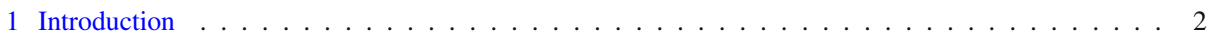

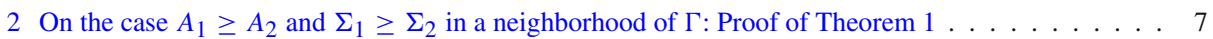

3 On the case $A_{1} \geq A_{2}$ and $\Sigma_{1}$ not greater than $\Sigma_{2}$ in a neighborhood of $\Gamma \ldots \ldots \ldots \ldots$

3.1 On the case $A_{1} \geq A_{2}$ and $\Sigma_{1} \leq \Sigma_{2}$ in a neighborhood of $\Gamma$-Proof of Theorem $2 \ldots \ldots 16$

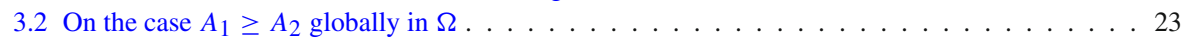

4 On the case $A_{1}=A_{2}$ in a neighborhood of $\Gamma$ : Proof of Theorem $3 \ldots \ldots \ldots \ldots$

5 On the case $A_{1}$ and $A_{2}$ satisfying the complementary condition on $\Gamma$ : Proof of Theorem $4 \ldots 32$

References . . . . . . . . . . . . . . . . . . . . . . . . 37

Mathematics Subject Classification 35P25 - 49R05 $\cdot$ 78A40 $\cdot 78 \mathrm{M} 30$

Communicated by P. Rabinowitz.

Hoai-Minh Nguyen

hoai-minh.nguyen@epfl.ch

Quoc-Hung Nguyen

quoc-hung.nguyen@epfl.ch

1 École Polytechnique Fédérale de Lausanne, SB MATHAA CAMA, Station 8, 1015 Lausanne, Switzerland 


\section{Introduction}

Let $d \geq 2$ and $\Omega$ be a smooth bounded simply connected open subset of $\mathbb{R}^{d}$ and denote $\Gamma=\partial \Omega$. Let $A_{1}, A_{2}$ be two real symmetric matrix-valued functions and $\Sigma_{1}, \Sigma_{2}$ be two bounded positive functions all defined in $\Omega$ such that $A_{1}$ and $A_{2}$ are uniformly elliptic and $\Sigma_{1}, \Sigma_{2}$ are bounded below by a positive constant in $\Omega$, i.e., for some constant $\Lambda \geq 1$, and for $j=1,2$,

$$
\Lambda^{-1}|\xi|^{2} \leq\left\langle A_{j}(x) \xi, \xi\right\rangle \leq \Lambda|\xi|^{2} \text { for all } \xi \in \mathbb{R}^{d} \text {, for a.e. } x \in \Omega,
$$

and

$$
\Lambda^{-1} \leq \Sigma_{j}(x) \leq \Lambda \text { for a.e. } x \in \Omega .
$$

Here and in what follows $\langle\cdot, \cdot\rangle$ denotes the Euclidean scalar product. A complex number $\lambda$ is called an eigenvalue of the interior transmission eigenvalue (ITE) problem associated with the pairs $\left(A_{1}, \Sigma_{1}\right)$ and $\left(A_{2}, \Sigma_{2}\right)$ in $\Omega$ if there is a non-zero pair of functions $\left(u_{1}, u_{2}\right) \in\left[H^{1}(\Omega)\right]^{2}$ satisfying the system

$$
\begin{cases}\operatorname{div}\left(A_{1} \nabla u_{1}\right)-\lambda \Sigma_{1} u_{1}=0 & \text { in } \Omega \\ \operatorname{div}\left(A_{2} \nabla u_{2}\right)-\lambda \Sigma_{2} u_{2}=0 & \text { in } \Omega\end{cases}
$$

and the boundary conditions

$$
u_{1}=u_{2}, \quad A_{1} \nabla u_{1} \cdot v=A_{2} \nabla u_{2} \cdot v \text { on } \Gamma,
$$

where $v$ denotes the outward normal unit vector on $\Gamma$. Such a pair $\left(u_{1}, u_{2}\right)$ is called an eigenfunction pair of (1.3) and (1.4).

The ITE problem was introduced in the middle of eighties by Kirsch [13] and Colton and Monk [7]. One of its interests comes from the connection between the density of the range of the far field operator with the injectivity of ITE problem. The ITE problem is known to be not self-adjoint. More severely, a priori estimates are non-standard and do not hold in general. These create many difficulties for the investigation and a source of interesting problems. Some important directions in the study of the ITE problems are the discreteness of the spectrum [5,8-10,13-16,28,29], the completeness of the generalized eigenfunctions $[2,16]$, and the Weyl-laws for the spectrum $[10,12,15,16]$. The reader can find a review on the ITE problem in [6].

This paper is devoted to the discreteness of the eigenvalues for the ITE problem. The discreteness of the spectrum of (1.3)-(1.4) was established by Rynne and Sleeman [28] and Colton et al. [8] in the case $A_{1}=A_{2}=I$, the identity matrix, $\Sigma_{1}-\Sigma_{2}=\Sigma_{1}-1 \geq c>0$ in $\Omega$ for some constant $c$. Using the fact that $\Sigma_{1}-\Sigma_{2}>c>0$, they can transform (1.3)-(1.4) into an elliptic equation of fourth order and then derive the discreteness of the spectrum of the ITE problem from the new one. The discreteness of the spectrum of the ITE problem involving only the information near the boundary was established quite recently. Using the T-coercivity method, which is related to the (Banach-Necas-Babuska) inf-sup condition, Bonnet-BenDhia et al. [3] obtained the discreteness of the spectrum of the ITE problem under the two assumptions $i)\left(A_{1}, \Sigma_{1}\right)=(I, 1)$ or $\left(A_{2}, \Sigma_{2}\right)=(I, 1)$ and $\left.i i\right)$ $A_{1}-A_{2} \geq c I$ and $\Sigma_{1}-\Sigma_{2} \geq c$ in a neighborhood of $\Gamma$ for some positive constant c. In [29], Sylvester showed that the discreteness takes place if $A_{1}=A_{2}=I$ in $\Omega$ and $\Sigma_{1}-\Sigma_{2}=\Sigma_{1}-1 \geq c>0$ in a neighbourhood of $\Gamma$ for some constant $c$ using the theory of upper triangular compact operators and an apriori estimate for the ITE problem. His method also works in the case $A_{1}=A_{2}$ smooth in $\Omega$. In [14], Lakshtanov and Vainberg obtained the discreteness of ITE problem under the assumption that $\left(A_{1}, \Sigma_{1}\right)=(I, 1),\left(A_{1}, A_{2}, \Sigma_{1}, \Sigma_{2}\right)$ is smooth in $\Omega$ and satisfies the so-called parameter-elliptic conditions. In a related direction, 
Lakshtanov and Vainberg [15] obtained the discreteness of the ITE problem for the case $\left(A_{1}, A_{2}, \Sigma_{1}, \Sigma_{2}\right)=\left(I, I, 1, \Sigma_{2}\right)$ in $\Omega, \Sigma_{2}$ is smooth in $\bar{\Omega}$, and $\Sigma_{2}=1$ and $\partial_{\nu} \Sigma_{2} \neq 0$ on $\Gamma$.

In this paper, we follow the standard strategy used in the context of Laplace operator to investigate the discreteness of the eigenvalues for the ITE problem. To this end, we first establish the well-posedness of the following system, for some $\gamma_{0} \in \mathbb{C}$,

$$
\begin{cases}\operatorname{div}\left(A_{1} \nabla u_{1}\right)-\gamma_{0} \Sigma_{1} u_{1}=g_{1} & \text { in } \Omega, \\ \operatorname{div}\left(A_{2} \nabla u_{2}\right)-\gamma_{0} \Sigma_{2} u_{2}=g_{2} & \text { in } \Omega, \\ u_{1}=u_{2}, \quad A_{1} \nabla u_{1} \cdot v=A_{2} \nabla u_{2} \cdot v & \text { on } \Gamma,\end{cases}
$$

where $\left(g_{1}, g_{2}\right)$ is a given pair of functions in an appropriate space. We then define the operator

$$
\begin{aligned}
T\left(f_{1}, f_{2}\right) & =\left(u_{1}, u_{2}\right) \text { where }\left(u_{1}, u_{2}\right) \text { is the unique solution of }(1.5) \text { with } \\
\left(g_{1}, g_{2}\right) & =\left(\Sigma_{1} f_{1}, \Sigma_{2} f_{2}\right) .
\end{aligned}
$$

The discreteness of the eigenvalues for the ITE problem can be now derived from the discreteness of the spectrum of $T$. In this paper, the well-posedness and the compactness of the operator $T$, for some appropriate choice $\gamma_{0} \in \mathbb{C}$ are obtained via the multiplier technique and the Fourier analysis. The first approach has roots from the work of Nguyen [23] where he studies the well-posedness of the Helmholtz equations with sign changing coefficients. Various ideas in this paper were introduced there. The Fourier analysis is quite natural in this setting and the idea is to compute solutions of Cauchy problems in half space and derive the information from this.

Throughout this paper

$$
d_{\Gamma}(x):=\operatorname{dist}(x, \Gamma):=\inf _{y \in \Gamma}|x-y| \text { for } x \in \mathbb{R}^{d},
$$

and for two matrices $M_{1}, M_{2}$, we denote $M_{1} \geq M_{2}$ if $M_{1}-M_{2}$ is a nonnegative matrix.

Our first result on the discreteness of the eigenvalues for the ITE problem is

Theorem 1 Assume that for some $0 \leq \alpha<2$ and for some positive constant $c$,

$$
A_{1}-A_{2} \geq c d_{\Gamma}^{\alpha} I \quad \text { and } \quad \Sigma_{1}-\Sigma_{2} \geq 0 \quad \text { in a neighborhood of } \Gamma .
$$

The spectrum of (1.3)-(1.4) is discrete.

No regularity assumption on $\left(A_{1}, A_{2}, \Sigma_{1}, \Sigma_{2}\right)$ is imposed in Theorem 1 . The conditions in (1.8) are only required near $\Gamma$ and neither upper bound of $A_{1}-A_{2}$ nor the one of $\Sigma_{1}-\Sigma_{2}$ is required. The proof of Theorem 1 is based on the multiplier technique and given in Sect. 2 . To our knowledge, Theorem 1 is new even in the case $\alpha=0$. Applying Theorem 1 for $\alpha=0$, one recovers and extends the discreteness results obtained by Bonnet-BenDhia et al. [3] mentioned.

When the second inequality in (1.8) is reversed and $\Sigma_{2}$ is large enough, the discreteness also holds. More precisely, we have

Theorem 2 Assume that for some $0 \leq \alpha<2$ and for some positive constant $c$,

$$
A_{1}-A_{2} \geq c d_{\Gamma}^{\alpha} I \quad \text { in a neighborhood of } \Gamma .
$$

Let $\Lambda_{1} \geq 1$ be such that

$$
\Lambda_{1}^{-1} I \leq A_{j} \leq \Lambda_{1} I \quad \text { for } j=1,2, \quad \text { and } \quad \Lambda_{1}^{-1} \leq \Sigma_{1} \leq \Lambda_{1} \quad \text { in } \Omega .
$$


For every $0<\Lambda_{2}<1$ there exists $K\left(\Lambda_{2}\right)>0$ depending only on $\Lambda_{1}, \Lambda_{2}, c, \alpha$, and the neighborhood of $\Gamma$ in (1.9) such that if

$$
\Lambda_{2} K \leq \Sigma_{2} \leq K \text { in } \Omega,
$$

for some $K>K\left(\Lambda_{2}\right)$, then the spectrum of (1.3)-(1.4) is discrete.

As far as we know, Theorem 2 is new even in the case $\alpha=0$. The proof of Theorem 2 is based on the multiplier technique and presented in Sect. 3. Another related result of Theorem 2 assuming only a mild condition on $\left(\Sigma_{1}, \Sigma_{2}\right)$ is given in Proposition 8; however, one requires that $A_{1}-A_{2} \geq c d_{\Gamma}^{\alpha} I$ in the whole domain $\Omega$.

We next deal with the case $A_{1}=A_{2}$ in a neighborhood of $\Gamma$.

Theorem 3 Assume that for some $0 \leq \beta<2$ and for some positive constants $c$,

$$
A_{1}=A_{2} \quad \text { and } \quad \Sigma_{1}-\Sigma_{2} \geq c d_{\Gamma}^{\beta} \text { in a neighborhood of } \Gamma \text {. }
$$

The spectrum of (1.3)-(1.4) is discrete.

Applying Theorem 3 for the case $A_{1}=A_{2}$ smooth in $\Omega$, and $\beta=0,1$, one recovers and extends the results mentioned of Sylvester [29] and Lakshtanov and Vainberg [15]. In comparison with their results, the novelty of Theorem 3 relies on the two facts: $i$ ) only the information near the boundary on $\left(A_{1}, A_{2}, \Sigma_{1}, \Sigma_{2}\right)$ is assumed and $\left.i i\right)$ no regularity on the coefficients is required.

In the case $\left(A_{1}, A_{2}, \Sigma_{1}, \Sigma_{2}\right)$ is continuous in a neighborhood of $\Gamma$, we prove the following result which involves the complementing condition due to Agmon et al. [1] (see also [17]):

Theorem 4 Assume that $A_{1}, A_{2}, \Sigma_{1}, \Sigma_{2}$ are continuous in a neighbourhood of $\Gamma$ and the following two conditions hold:

(i) For all $x \in \Gamma, A_{1}(x), A_{2}(x)$ satisfy the complementing boundary condition with respect to $v(x)$,

(ii) For all $x \in \Gamma,\left\langle A_{1}(x) v(x), v(x)\right| \Sigma_{1}(x) \neq\left\langle A_{2}(x) v(x), v(x)\right| \Sigma_{2}(x)$.

The spectrum of (1.3)-(1.4) is discrete.

The complementing condition corresponding to the ITE problem has the following explicit characterization given in [23, Proposition 1] (see e.g. [23, Definition 2] for the definition of the complementing condition for the ITE problem).

Proposition 1 Let e be a unit vector in $\mathbb{R}^{d}$ and let $A_{1}$ and $A_{2}$ be two constant positive symmetric matrices. Then $A_{1}$ and $A_{2}$ satisfy the complementing boundary condition with respect to $e$ if and only if

$$
\left\langle A_{2} e, e\right\rangle\left\langle A_{2} \xi, \xi\right\rangle-\left\langle A_{2} e, \xi\right\rangle^{2} \neq\left\langle A_{1} e, e\right\rangle\left\langle A_{1} \xi, \xi\right\rangle-\left\langle A_{1} e, \xi\right\rangle^{2} \forall \xi \in P \backslash\{0\},
$$

where

$$
\mathcal{P}:=\left\{\xi \in \mathbb{R}^{d} ;\langle\xi, e\rangle=0\right\} .
$$

We next give some comments on the conditions $i$ ) and $i i$ ) of Theorem 4 :

- It is shown in [23, Proposition 1] that if $A_{1}>A_{2}$ then $A_{1}$ and $A_{2}$ satisfy the complementing boundary condition for all unit vector $e$.

- It is clear from (1.13) that if $d=2$ then (1.13) is equivalent to the condition $\operatorname{det} A_{1} \neq$ $\operatorname{det} A_{2}$. 
- Assume that $A_{1}$ and $A_{2}$ are isotropic on $\Gamma$, i.e., $A_{1}=a_{1} I$ and $A_{2}=a_{2} I$ for some (positive) functions $a_{1}, a_{2}$ on $\Gamma$. Then $i$ ) and $i i$ ) are equivalent to the conditions $a_{1} \neq a_{2}$ and $a_{1} \Sigma_{1} \neq a_{2} \Sigma_{2}$ on $\Gamma$.

The proof of Theorem 4 is via Fourier analysis and given in Sect. 5. Applying Theorem 4 and Proposition 1, one recovers and extends the result of Lakshtanov and Vainberg [14].

The discreteness of the eigenvalues of a general elliptic system equipped complementing boundary condition was investigated by Faierman [10] and references therein. The conditions given in [10] are not explicit and require that the coefficients are of class $C^{1}(\bar{\Omega})$. The proof in [10] involved the work of Agmon et al. [1].

It is worth noting that ITE problem is invariant after a change of variables. In fact, let $F$ be a diffeomorphism from $\Omega$ into itself ${ }^{1}$ such that $F(x)=x$ on $\Gamma$. Set $U_{1}=u_{1} \circ F^{-1}$ in $\Omega$. By the rule of a change of variables (see e.g. [18, Lemma 2]), $u_{1} \in H^{1}(\Omega)$ is a solution to the equation

$$
\operatorname{div}\left(A_{1} \nabla u_{1}\right)-\lambda \Sigma_{1} u_{1}=0 \text { in } \Omega
$$

if and only if

$$
\operatorname{div}\left(F_{*} A_{1} \nabla U_{1}\right)-\lambda F_{*} \Sigma_{1} U_{1}=0 \text { in } \Omega,
$$

where

$$
F_{*} A_{1}(y)=\frac{D F(x) A_{1}(x) D F^{T}(x)}{\operatorname{det} D F(x)} \quad \text { and } \quad F_{*} \Sigma_{1}(y)=\frac{\Sigma_{1}(x)}{\operatorname{det} D F(x)} \text { with } x=F^{-1}(y) .
$$

Moreover,

$$
U_{1}=u_{1} \quad \text { and } \quad F_{*} A_{1} \nabla U_{1} \cdot v=A_{1} \nabla u_{1} \cdot v \text { on } \Gamma .
$$

It follows that $\left(u_{1}, u_{2}\right) \in\left[H^{1}(\Omega)\right]^{2}$ is a solution of (1.3)-(1.4) if and only if $\left(U_{1}, U_{2}\right)=$ $\left(u_{1} \circ F^{-1}, u_{2}\right) \in\left[H^{1}(\Omega)\right]^{2}$ is a solution of the system

$$
\begin{cases}\operatorname{div}\left(F_{*} A_{1} \nabla U_{1}\right)-\lambda F_{*} \Sigma_{1} U_{1}=0 & \text { in } \Omega, \\ \operatorname{div}\left(A_{2} \nabla U_{2}\right)-\lambda \Sigma_{2} U_{2}=0 & \text { in } \Omega, \\ U_{1}=U_{2}, \quad F_{*} A_{1} \nabla U_{1} \cdot v=A_{2} \nabla U_{2} \cdot v & \text { on } \Gamma .\end{cases}
$$

Moreover, $\left(u_{1}, u_{2}\right) \neq 0$ if and only if $\left(U_{1}, U_{2}\right) \neq 0$. Using this observation, we can extend the previous results in which the conditions on $\left(A_{1}, \Sigma_{1}\right)$ and $\left(A_{2}, \Sigma_{2}\right)$ are involved to the case where the same conditions hold for $\left(F_{*} A_{1}, F_{*} \Sigma_{1}\right)$ and $\left(A_{2}, \Sigma_{2}\right)$ for some diffeomorphism $F$ verifying the condition $F(x)=x$ on $\Gamma^{2}$. We state here two results following from Theorems 1 and 3 in this direction as an illustration. A variant of Theorem 2 is left to the reader. Theorem 4 is already invariant under a change of variables.

Proposition 2 Assume that for some diffeomorphisms $F$ from $\Omega$ into itself such that $F(x)=$ $x$ on $\Gamma$, and for $0 \leq \alpha<2$ and for some positive constant $c$,

$$
F_{*} A_{1}-A_{2} \geq c d_{\Gamma}^{\alpha} I \quad \text { and } \quad F_{*} \Sigma_{1}-\Sigma_{2} \geq 0 \quad \text { in a neighborhood of } \Gamma .
$$

The spectrum of (1.3)-(1.4) is discrete.

\footnotetext{
${ }^{1}$ In this paper, this means that $F$ is bijective and $F, F^{-1} \in C^{1}(\bar{\Omega})$.

2 One can use two diffeomorphisms $F_{1}, F_{2}$ and require the corresponding conditions on $\left(F_{1 *} A_{1}, F_{1 *} \Sigma_{1}\right)$ and $\left(F_{2 *} A_{2}, F_{2 *} \Sigma_{2}\right)$ to obtain the discreteness of the ITE problem. However, the same conditions hold by using the diffeomorphisms $F_{1} \circ F_{2}^{-1}, I$.
} 
Proposition 3 Assume that for some diffeomorphism $F$ from $\Omega$ into itself such that $F(x)=x$ on $\Gamma$,

$$
F_{*} A_{1}=A_{2} \quad \text { and } \quad F_{*} \Sigma_{1}-\Sigma_{2} \geq c d_{\Gamma}^{\beta} \quad \text { in a neighborhood of } \Gamma,
$$

for some $0 \leq \beta<2$ and for some positive constants $c$. The spectrum of (1.3)-(1.4) is discrete.

Applying Propositions 2 and 3, one is able to obtain the discreteness of the spectrum of the ITE problem even in the case $A_{1}-A_{2}$ changes the sign in a neighborhood of $\Gamma$.

We now describe briefly the ideas of the proof of Theorems 1,2,3, and 4. The proof of Theorems 1, 2, and 3 are based on the multiplier technique while the proof of Theorem 4 is based on the Fourier analysis. The key point is to establish the well-posedness and the compactness of $T$ given by (1.6) for some $\gamma_{0} \in \mathbb{C}$. Concerning Theorem 1 , the well-posedness of and the compactness of $T$ take place for $\gamma_{0}=\lambda_{0}$ for some large positive $\lambda_{0}$. The existence of a solution of the system (1.5) is based on a priori estimates (Lemma 1) for a Cauchy problem with its roots from [23] and via the limiting absorption process (Lemma 7). To require the information only near the boundary $\Gamma, \lambda_{0}$ is chosen to be large and a standard exponential decay estimate for elliptic equations is involved (Lemma 2). Since the exponent $\alpha$ might be positive, some weighted spaces are involved and the solutions are not in $\left[H^{1}(\Omega)\right]^{2}$ as usual. The proof of the uniqueness of (1.5) faces some issues due to the lack of the regularity of the solutions. To overcome this, we introduce the concept of viscosity solution (Definition 2), the terminology is inspired by the one of systems of conservation laws. The compactness of $T$ follows from the condition $0 \leq \alpha<2$ in (1.8). Concerning Theorem 2, the well-posedness of and the compactness of $T$ take place for $\gamma_{0}=i \lambda_{0}$ for some small positive $\lambda_{0}$. This modification is necessary for the proof of the uniqueness. As in the proof of Theorem 1, a priori estimates hold for (3.1) (Lemma 8). Nevertheless, the uniqueness of (3.1) even for smooth solutions does not follow directly from the a priori estimates as in the proof of Theorem 1. Additional arguments are required in this case (Lemma 10). Beside these points, the proof of Theorem 2 follows similarly as the one of Theorem 1 . The proof of Theorem 3 is somehow in the spirit of the one of Theorem 1 but with the following key difference. While the same arguments as used in the proof of Theorem 1 imply the uniqueness of $T$ with $\gamma_{0}=\lambda_{0}$ for large positive $\lambda_{0}$, they do not imply the compactness of $T$ since the first condition in (1.8) does not hold. To be able to deal with the situation in which $A_{1}=A_{2}$ in a neighborhood of $\Gamma$, we make some modifications on $T$. The idea is to take into account the fact from the a priori estimates $u_{1}-u_{2}$ is more regular than $u_{1}$ and $u_{2}$. After the modification of $T$, the proof of Theorem 3 is in the spirit of the one of Theorem 1 but the functional spaces used in this case is different and somehow more involved and the theory of compact analytic operator is used. The proof of Theorem 4 is via the Fourier analysis. The approach is based on the computation of solutions of the Cauchy problems in half space via Fourier analysis. We then use local charts and the exponential decays of the solutions to deal with the general case. The analysis is also in the spirit of the work Agmon et al. [1].

The analysis in this paper is devoted to the study of the Cauchy problem (1.3) and (1.4). There is a connection between the study of the Cauchy problem and the Helmholtz equations with sign changing coefficients modelling negative index materials. This connection was pointed out by Nguyen [23]. Various ideas in this paper were introduced there. It is worth noting that resonance might appear in various interesting potential applications of negative index materials such as superlensing using complementary media [19], cloaking using complementary media $[22,26]$, cloaking a source via anomalous localized resonance, see e.g. $[20,21,24]$, and cloaking an object via anomalous localized resonance in [25]. 
The paper is organized as follows. The proof of Theorems 1, 2, 3, and 4 are given in Sects. 2, 3, 4, and 5 respectively.

\section{On the case $A_{1} \geq A_{2}$ and $\Sigma_{1} \geq \Sigma_{2}$ in a neighborhood of $\Gamma$ : Proof of Theorem 1}

The section is devoted to the proof of Theorem 1 . To this end, as mentioned in the introduction, we first establish the well-posedness of the following system, for some $\lambda_{0}>0$,

$$
\begin{cases}\operatorname{div}\left(A_{1} \nabla u_{1}\right)-\lambda_{0} \Sigma_{1} u_{1}=g_{1} & \text { in } \Omega, \\ \operatorname{div}\left(A_{2} \nabla u_{2}\right)-\lambda_{0} \Sigma_{2} u_{2}=g_{2} & \text { in } \Omega, \\ u_{1}=u_{2}, \quad A_{1} \nabla u_{1} \cdot v=A_{2} \nabla u_{2} \cdot v & \text { on } \Gamma,\end{cases}
$$

for a given pair $\left(g_{1}, g_{2}\right)$ in an appropriate space. We then define the operator

$$
\begin{aligned}
T_{1}\left(f_{1}, f_{2}\right) & =\left(u_{1}, u_{2}\right) \text { where }\left(u_{1}, u_{2}\right) \text { is the unique solution of }(2.1) \\
\text { with }\left(g_{1}, g_{2}\right) & =\left(\Sigma_{1} f_{1}, \Sigma_{2} f_{2}\right)
\end{aligned}
$$

and prove the compactness for it.

The proof of the well-posedness of (2.1) is as follows. The existence of a solution of (2.1) is based on a priori estimates (Lemma 1) for a Cauchy problem with roots from [23] and via a limiting absorption process (Lemma 7). To require only the information near the boundary, $\lambda_{0}$ is chosen to be large and a standard exponential decay estimate for elliptic equations is involved (Lemma 2). The solutions are not in $\left[H^{1}(\Omega)\right]^{2}$ as usual and hence it is not clear whether the a priori estimates hold for the difference of two solutions. To overcome this issue, we introduce the concept of viscosity solutions (Definition 2). We now present the details of the proof. We start with the following result (Lemma 1) which plays an important role in the proof of Theorem 1. Lemma 1 is a more detailed version of [23, Lemma 9] and its proof follows closely from there.

Lemma 1 Let $g=\left(g_{1}, g_{2}\right) \in\left[L^{2}(\Omega)\right]^{2}, h \in H^{-1 / 2}(\Gamma)$, and $\lambda_{0} \geq 0$, and let $v=\left(v_{1}, v_{2}\right) \in$ $\left[H^{1}(\Omega)\right]^{2}$ satisfy

$$
\begin{cases}\operatorname{div}\left(A_{1} \nabla v_{1}\right)-\lambda_{0} \Sigma_{1} v_{1}=g_{1} & \text { in } \Omega, \\ \operatorname{div}\left(A_{2} \nabla v_{2}\right)-\lambda_{0} \Sigma_{2} v_{2}=g_{2} & \text { in } \Omega, \\ v_{1}=v_{2}, \quad A_{1} \nabla v_{1} \cdot v=A_{2} \nabla v_{2} \cdot v+h & \text { on } \Gamma\end{cases}
$$

We have, with $w=v_{1}-v_{2}$,

$$
\begin{aligned}
\int_{\Omega}\left\langle A_{1} \nabla w, \nabla w\right\rangle+\lambda_{0} \Sigma_{1}|w|^{2}= & -\int_{\Omega}\left(g_{1}-g_{2}\right) \bar{w}+\int_{\Omega} \lambda_{0}\left(\Sigma_{2}-\Sigma_{1}\right) v_{2} \bar{w} \\
& +\int_{\Omega}\left\langle\left[A_{2}-A_{1}\right] \nabla v_{2}, \nabla w\right\rangle
\end{aligned}
$$

and

$$
\begin{aligned}
& \int_{\Omega}\left\langle\left[A_{1}-A_{2}\right] \nabla v_{2}, \nabla w\right\rangle+\left\langle\left[A_{1}-A_{2}\right] \nabla v_{2}, \nabla v_{2}\right\rangle+\lambda_{0}\left(\Sigma_{1}-\Sigma_{2}\right)\left|v_{2}\right|^{2} \\
& \quad=\int_{\Omega} g_{2} \bar{w}-\left(\bar{g}_{1}-\bar{g}_{2}\right) v_{2}+\lambda_{0}\left(\Sigma_{2}-\Sigma_{1}\right) v_{2} \bar{w}+\int_{\Gamma} \bar{h} v_{2} .
\end{aligned}
$$


As a consequence of (2.4) and (2.5), we obtain

$$
\int_{\Omega}\left\langle A_{1} \nabla w, \nabla w\right\rangle+\lambda_{0} \Sigma_{1}|w|^{2} \leq 4 \mathcal{M}(v, g, h)+\int_{\Omega}\left\langle\left[A_{2}-A_{1}\right] \nabla v_{2}, \nabla w\right\rangle+\lambda_{0}\left(\Sigma_{2}-\Sigma_{1}\right) v_{2} \bar{w},
$$

and

$$
\begin{aligned}
& \int_{\Omega}\left\langle\left[A_{1}-A_{2}\right] \nabla v_{2}, \nabla v_{2}\right\rangle+\lambda_{0}\left(\Sigma_{1}-\Sigma_{2}\right)\left|v_{2}\right|^{2} \\
& \quad \leq 4 \mathcal{M}(v, g, h)+\int_{\Omega}\left|\left\langle\left[A_{2}-A_{1}\right] \nabla v_{2} \nabla w\right\rangle\right|+\lambda_{0}\left|\left(\Sigma_{2}-\Sigma_{1}\right) v_{2} \bar{w}\right|,
\end{aligned}
$$

where

$$
\mathcal{M}(v, g, h):=\|v\|_{L^{2}(\Omega)}\|g\|_{L^{2}(\Omega)}+\|h\|_{H^{-1 / 2}(\Gamma)}\|v\|_{H^{1 / 2}(\Gamma)} .
$$

Here and in what follows in this paper, for a complex number $z, \bar{z}$ denotes its conjugate.

Proof We derive from the definition of $w$ that $w=0$ on $\Gamma$ and

$$
\operatorname{div}\left(A_{1} \nabla w\right)-\lambda_{0} \Sigma_{1} w=g_{1}-g_{2}-\lambda_{0}\left(\Sigma_{2}-\Sigma_{1}\right) v_{2}+\operatorname{div}\left(\left[A_{2}-A_{1}\right] \nabla v_{2}\right) \text { in } \Omega .
$$

Multiplying this equation by $\bar{w}$ and integrating on $\Omega$, we have

$$
\begin{aligned}
\int_{\Omega}\left\langle A_{1} \nabla w, \nabla w\right\rangle+\lambda_{0} \Sigma_{1}|w|^{2}= & -\int_{\Omega}\left(g_{1}-g_{2}\right) \bar{w}+\int_{\Omega} \lambda_{0}\left(\Sigma_{2}-\Sigma_{1}\right) v_{2} \bar{w} \\
& +\int_{\Omega}\left\langle\left[A_{2}-A_{1}\right] \nabla v_{2}, \nabla w\right\rangle ;
\end{aligned}
$$

which is (2.4). Multiplying the equation of $v_{2}$ by $\bar{w}$ and integrating on $\Omega$, we obtain

$$
\int_{\Omega}-\left\langle A_{2} \nabla v_{2}, \nabla w\right\rangle-\lambda_{0} \Sigma_{2} v_{2} \bar{w}=\int_{\Omega} g_{2} \bar{w}
$$

It is clear that

$$
-A_{2} \nabla w=\left(A_{1}-A_{2}\right) \nabla w-A_{1} \nabla w+\left(A_{2}-A_{1}\right) \nabla v_{2}+\left(A_{1}-A_{2}\right) \nabla v_{2} \text { in } \Omega,
$$

and, by (2.9),

$$
\operatorname{div}\left(A_{1} \nabla w\right)-\operatorname{div}\left(\left[A_{2}-A_{1}\right] \nabla v_{2}\right)=\lambda_{0} \Sigma_{1} w+g_{1}-g_{2}-\lambda_{0}\left(\Sigma_{2}-\Sigma_{1}\right) v_{2} \text { in } \Omega .
$$

Since

$$
\left(A_{1} \nabla w-\left(A_{2}-A_{1}\right) \nabla v_{2}\right) \cdot v=\left(A_{1} \nabla v_{1}-A_{2} \nabla v_{2}\right) \cdot v=h \text { on } \Gamma,
$$

it follows from (2.10), (2.11), and (2.12) that

$$
\begin{aligned}
& \int_{\Omega}\left\langle\left[A_{1}-A_{2}\right] \nabla v_{2}, \nabla w\right\rangle+\left\langle\left[A_{1}-A_{2}\right] \nabla v_{2}, \nabla v_{2}\right\rangle+\lambda_{0}\left(\Sigma_{1}-\Sigma_{2}\right)\left|v_{2}\right|^{2} \\
& \quad=\int_{\Omega} g_{2} \bar{w}-\left(\bar{g}_{1}-\bar{g}_{2}\right) v_{2}+\lambda_{0}\left(\Sigma_{2}-\Sigma_{1}\right) v_{2} \bar{w}+\int_{\Gamma} \bar{h} v_{2} ;
\end{aligned}
$$

which is (2.5).

Assertion (2.6) and (2.7) are direct consequences of (2.4) and (2.5). The proof is complete. 
In what follows, we denote, for $s>0$,

$$
\Omega_{s}:=\left\{x \in \Omega ; d_{\Gamma}(x)<s\right\} .
$$

The following exponential decay property for elliptic equations is useful for our analysis.

Lemma 2 Let $\lambda>1, f \in L^{2}(\Omega)$, A be a matrix-valued function, and $\Sigma$ be real function defined in $\Omega$ such that, for some $\Lambda>1$,

$$
\Lambda^{-1} I \leq A \leq \Lambda I \quad \text { and } \quad \Lambda^{-1} \leq \Sigma \leq \Lambda \text { in } \Omega
$$

Let $u \in H_{\text {loc }}^{1}(\Omega)$ be a solution to the equation $\operatorname{div}(A \nabla u)-\lambda \Sigma u=f$ in $\Omega$. For all $s>0$, there exist two positive constants $c_{1}$ and $c_{2}$, depending only on $\Lambda, s$, and $\Omega$, such that

$$
\|u\|_{H^{1}\left(\Omega \backslash \Omega_{s}\right)} \leq c_{1} \exp \left(-c_{2} \sqrt{\lambda}\right)\|u\|_{L^{2}\left(\Omega_{s}\right)}+c_{1}\|f\|_{L^{2}(\Omega)} .
$$

Proof Lemma 2 is quite standard and its proof presented here is in the spirit of the one of [11, Theorem 2.2]. Let $U \in H_{0}^{1}(\Omega)$ be the unique solution of the equation $\operatorname{div}(A \nabla U)-\lambda \Sigma U=f$ in $\Omega$. Then $\|U\|_{H^{1}(\Omega)} \leq C\|f\|_{L^{2}(\Omega)}$. Here and in what follows in this proof $C$ denotes a positive constant depending only on $\Lambda, s$, and $\Omega$. By considering $u-U$, without loss of generality, one might assume that $f=0$ in $\Omega$; this is assumed from now. Fix $\varphi \in C^{2}(\Omega)$ such that $\varphi=c s$ in $\Omega \backslash \Omega_{s}$ and $\varphi=0$ in $\Omega_{s / 2}$, and $|\nabla \varphi| \leq c$ in $\Omega$ where $c$ is a small positive constant defined later (the smallness of $c$ depends only on $\Lambda$ and $\Omega$, it is independent of $s$ ). Set $\phi(x)=e^{\sqrt{\lambda} \varphi(x)}$ and $v(x)=u(x) \phi(x)$ for $x \in \Omega$. Since $\operatorname{div}(A \nabla u)-\lambda \Sigma u=0$ in $\Omega$, it follows that

$$
\operatorname{div}(A \nabla v)-\lambda \Sigma v=\sqrt{\lambda} \operatorname{div}(v A \nabla \varphi)+\sqrt{\lambda} A \nabla v \nabla \varphi-\lambda v A \nabla \varphi \nabla \varphi \text { in } \Omega .
$$

Note that, by Cacciopoli's inequality,

$$
\|u\|_{H^{1}\left(\Omega_{s / 2} \backslash \Omega_{s / 4}\right)} \leq C \sqrt{\lambda}\|u\|_{L^{2}\left(\Omega_{s}\right)} .
$$

Hence there exists $\tau \in(s / 4, s / 2)$ such that $\|u\|_{H^{1}\left(\partial\left(\Omega \backslash \Omega_{\tau}\right)\right)} \leq C \sqrt{\lambda}\|u\|_{L^{2}\left(\Omega_{s}\right)}$. Multiplying (2.15) by $\bar{v}$, integrating by parts in $\Omega \backslash \Omega_{\tau}$, and using (2.13) and the fact that $\varphi=0$ in $\Omega_{s / 2}$, we have

$$
\int_{\Omega \backslash \Omega_{\tau}}|\nabla v|^{2}+\lambda|v|^{2} \leq C\left(\int_{\Omega_{\backslash \Omega_{\tau}}} \sqrt{\lambda}|v||\nabla v \| \nabla \varphi|+\lambda|v|^{2}|\nabla \varphi|^{2}\right)+C \lambda\|u\|_{L^{2}\left(\Omega_{s}\right)}^{2} .
$$

By taking $c$ small enough, one can absorb the first term in the RHS of (2.16) by the LHS, and the conclusion follows.

We next recall the following compactness result from $[23 \text {, Lemma } 7]^{3}$.

Lemma 3 Let $0 \leq \alpha<2$ and $\left(u_{n}\right) \subset H_{l o c}^{1}(\Omega)$. Assume that

$$
\sup _{n} \int_{\Omega}\left(d_{\Gamma}^{\alpha}\left|\nabla u_{n}\right|^{2}+\left|u_{n}\right|^{2}\right) d x<+\infty
$$

Then $\left(u_{n}\right)$ is relatively compact in $L^{2}(\Omega)$.

Using Lemma 3, we can prove

3 In fact, [23, Lemma 7] is stated for $\left(u_{n}\right) \subset H^{1}(\Omega)$, however the result also holds for $\left(u_{n}\right) \subset H_{l o c}^{1}(\Omega)$ and the proof is almost unchanged. 
Lemma 4 Let $\sigma$ be a bounded real function defined in $\Omega$ such that $\int_{\Omega} \sigma \neq 0$. There exists a positive constant $C$ such that

$$
\int_{\Omega}|u|^{2} \leq C\left(\left.\left.\left|\int_{\Omega} \sigma\right| u\right|^{2}\left|+\int_{\Omega} d_{\Gamma}^{\alpha}\right| \nabla u\right|^{2}\right) \quad \forall u \in H_{l o c}^{1}(\Omega) .
$$

Proof Lemma 4 can be derived from Lemma 3 by a contradiction argument as follows. Assume that (2.18) does not hold. There exists a sequence $\left(u_{n}\right) \subset H_{l o c}^{1}(\Omega)$ such that

$$
n\left(\left.\left.\left|\int_{\Omega} \sigma\right| u_{n}\right|^{2}\left|+\int_{\Omega} d_{\Gamma}^{\alpha}\right| \nabla u_{n}\right|^{2}\right) \leq \int_{\Omega}\left|u_{n}\right|^{2}=1 .
$$

By Lemma 3, one may assume that $u_{n} \rightarrow u$ strongly in $L^{2}(\Omega)$ and almost everywhere in $\Omega$, and $u_{n} \rightarrow u$ weakly in $H_{l o c}^{1}(\Omega)$. We deduce from (2.19) that

$$
\left.\left.\left|\int_{\Omega} \sigma\right| u\right|^{2}\left|+\int_{\Omega} d_{\Gamma}^{\alpha}\right| \nabla u\right|^{2}=0 \quad \text { and } \quad \int_{\Omega}|u|^{2}=1 .
$$

The first identity implies that $u=0$ in $\Omega$ since $\Omega$ is connected; this contradicts the second identity. Hence (2.18) holds.

Using Lemma 2, we obtain

Lemma 5 Let $0 \leq \alpha<2$ and assume that, for some $\tau>0$ and $c>0$,

$$
A_{1}-A_{2} \geq c d_{\Gamma}^{\alpha} I \quad \text { and } \quad \Sigma_{1}-\Sigma_{2} \geq 0 \text { in } \Omega_{\tau} .
$$

There exists $\Lambda_{0}>1$ such that if $\lambda_{0} \geq \Lambda_{0}, v=\left(v_{1}, v_{2}\right) \in\left[H_{l o c}^{1}(\Omega)\right]^{2}$ satisfies the system

$$
\left\{\begin{array}{l}
\operatorname{div}\left(A_{1} \nabla v_{1}\right)-\lambda_{0} \Sigma_{1} v_{1}=g_{1} \text { in } \Omega, \\
\operatorname{div}\left(A_{2} \nabla v_{2}\right)-\lambda_{0} \Sigma_{2} v_{2}=g_{2} \text { in } \Omega,
\end{array}\right.
$$

and (2.6) and (2.7) hold for some $g=\left(g_{1}, g_{2}\right) \in\left[L^{2}(\Omega)\right]^{2}$ and $h \in H^{-1 / 2}(\Gamma)$, then, with $w=v_{1}-v_{2}$,

$$
\int_{\Omega_{\tau}}\left\langle\left[A_{1}-A_{2}\right] \nabla v_{2}, \nabla v_{2}\right\rangle+\int_{\Omega \backslash \Omega_{\tau}}\left|\nabla v_{2}\right|^{2}+\int_{\Omega}|\nabla w|^{2}+\left|v_{2}\right|^{2} \leq C\left(\mathcal{M}(v, g, h)+\|g\|_{L^{2}(\Omega)}^{2}\right),
$$

for some positive constant $C$ independent of $v, g$, and $h$ where $\mathcal{M}(v, g, h)$ is defined by (2.8). In particular if $v \in\left[H^{1}(\Omega)\right]^{2}$ is a solution of (2.3) then (2.22) holds.

Proof Since $A_{1}-A_{2} \geq 0$ and $\Sigma_{1}-\Sigma_{2} \geq 0$ in $\Omega_{\tau}$, we have, for all $\gamma>0$,

$\gamma \int_{\Omega_{\tau}}\left\langle\left[A_{1}-A_{2}\right] \nabla w, \nabla w\right\rangle+\frac{1}{\gamma} \int_{\Omega_{\tau}}\left\langle\left[A_{1}-A_{2}\right] \nabla v_{2}, \nabla v_{2}\right\rangle \geq 2 \int_{\Omega_{\tau}}\left|\left\langle\left[A_{1}-A_{2}\right] \nabla w, \nabla v_{2}\right\rangle\right|$

and

$$
\gamma \int_{\Omega_{\tau}}\left(\Sigma_{1}-\Sigma_{2}\right)|w|^{2}+\frac{1}{\gamma} \int_{\Omega_{\tau}}\left(\Sigma_{1}-\Sigma_{2}\right)\left|v_{2}\right|^{2} \geq 2 \int_{\Omega_{\tau}}\left(\Sigma_{1}-\Sigma_{2}\right)\left|w v_{2}\right| .
$$

Taking $\gamma>1$ and close to 1 , and adding (2.6) and (2.7), we derive that

$$
\begin{aligned}
& \int_{\Omega_{\tau}}\langle\nabla w, \nabla w\rangle+\lambda_{0}|w|^{2}+\left\langle\left[A_{1}-A_{2}\right] \nabla v_{2}, \nabla v_{2}\right\rangle \\
& \quad \leq C \mathcal{M}(v, g, h)+C \int_{\Omega \backslash \Omega_{\tau}}\left|\nabla v_{2}\right||\nabla w|+\lambda_{0}\left|v_{2}\right||w|+\left|\nabla v_{2}\right|^{2}+\lambda_{0}\left|v_{2}\right|^{2} .
\end{aligned}
$$


Here and in what follows in this proof, $C$ denotes a positive constant independent of $v, g, h$ and $\lambda_{0}$.

On the other hand, applying Lemma 6 below, we have

$$
\int_{\Omega} \lambda_{0} d_{\Gamma}^{\alpha+2}\left|v_{2}\right|^{2} \leq C \int_{\Omega} d_{\Gamma}^{\alpha}\left|\nabla v_{2}\right|^{2}+C\left\|g_{2}\right\|_{L^{2}(\Omega)}^{2} .
$$

This implies, by applying Lemma 4 with $\sigma=d_{\Gamma}^{\alpha+2}$,

$$
\int_{\Omega}\left|v_{2}\right|^{2} \leq C \int_{\Omega} d_{\Gamma}^{\alpha}\left|\nabla v_{2}\right|^{2}+C\left\|g_{2}\right\|_{L^{2}(\Omega)}^{2} .
$$

The conclusion now follows from (2.25) and (2.26) by applying Lemma 2 to $v_{2}$ and taking $\Lambda_{0}$ large enough.

Remark 1 Using (2.26), one can weaken the assumption $\Sigma_{1}-\Sigma_{2} \geq 0$ in (2.20) by $\Sigma_{1}-\Sigma_{2} \geq$ $-\hat{c}$ in a neighborhood of $\Gamma$ for some small positive constant $\hat{c}$.

In the proof of Lemma 5, we used the following result.

Lemma 6 Let $\lambda>1, \alpha \geq 0, f \in L^{2}(\Omega), A$ be a matrix-valued function, and $\Sigma$ be real function defined in $\Omega$ such that

$$
\Lambda^{-1} I \leq A \leq \Lambda I \quad \text { and } \quad \Lambda^{-1} \leq \Sigma \leq \Lambda \text { in } \Omega .
$$

Let $u \in H_{\text {loc }}^{1}(\Omega)$ be a solution to the equation $\operatorname{div}(A \nabla u)-\lambda \Sigma u=f$ in $\Omega$. We have

$$
\int_{\Omega} \lambda d_{\Gamma}^{\alpha+2}|u|^{2} \leq C \int_{\Omega} d_{\Gamma}^{\alpha}|\nabla u|^{2}+C\|f\|_{L^{2}(\Omega)}^{2}
$$

and

$$
\int_{\Omega} d_{\Gamma}^{\alpha+2}|\nabla u|^{2} \leq C \int_{\Omega} \lambda d_{\Gamma}^{\alpha}|u|^{2}+C\|f\|_{L^{2}(\Omega)}^{2},
$$

for some positive constant $C$ independent of $\lambda, f$, and $u$.

Proof We only prove (2.27); the proof of (2.28) follows similarly. Set $\Gamma_{s}=\partial\left(\Omega \backslash \Omega_{s}\right)$ and $\varphi_{s}(x)=\operatorname{dist}\left(x, \Gamma_{s}\right)^{\alpha / 2+1}$. Multiplying the equation of $u$ by $\bar{u} \varphi_{s}^{2}$ and integrating in $\Omega \backslash \Omega_{s}$, we have

$$
\int_{\Omega \backslash \Omega_{s}} \lambda\left|u \varphi_{s}\right|^{2}+\int_{\Omega \backslash \Omega_{s}}|\nabla u|^{2} \varphi_{s}^{2} \leq C \int_{\Omega \backslash \Omega_{s}}|\nabla u|\left|\nabla \varphi_{s}\right|\left|u \varphi_{s}\right|+|f|\left|u \varphi_{s}^{2}\right| .
$$

Since $|\nabla u|\left|\nabla \varphi_{s}\right|\left|u \varphi_{s}\right| \leq \gamma\left|\nabla \varphi_{s}\right|^{2}|\nabla u|^{2}+\frac{1}{4 \gamma}\left|\varphi_{s}\right|^{2}|u|^{2} \leq C \gamma \operatorname{dist}^{\alpha}\left(x, \Gamma_{s}\right)|\nabla u|^{2}+$ $\frac{1}{4 \gamma}\left|\varphi_{s}\right|^{2}|u|^{2}$ (for $\gamma>0$ ), it follows that

$$
\int_{\Omega \backslash \Omega_{s}} \lambda\left|u \varphi_{s}\right|^{2} \leq C \int_{\Omega \backslash \Omega_{s}} \operatorname{dist}\left(x, \Gamma_{s}\right)^{\alpha}|\nabla u|^{2}+|f|^{2} .
$$

Letting $s \rightarrow 0$, applying the Fatou lemma to the LHS and the dominated convergence theorem to the RHS, one obtains (2.27).

Remark 2 The same conclusion of Lemma 6 holds if instead of imposing that $\operatorname{div}(A \nabla u)-$ $\lambda \Sigma u=f$ in $\Omega$, one assumes $\operatorname{div}(A \nabla u)-i \lambda \Sigma u=f$ in $\Omega$.

The next basic result involving the limiting absorption process is 
Lemma 7 Let $\lambda_{0} \geq 0,0<\delta<1, g=\left(g_{1}, g_{2}\right) \in\left[L^{2}(\Omega)\right]^{2}$. There exists a unique solution $v_{\delta}=\left(v_{1, \delta}, v_{2, \delta}\right) \in\left[H^{1}(\Omega)\right]^{2}$ of the system

$$
\begin{cases}\operatorname{div}\left((1+i \delta) A_{1} \nabla v_{1, \delta}\right)-\lambda_{0} \Sigma_{1} v_{1, \delta}-i \delta v_{1, \delta}=g_{1} & \text { in } \Omega, \\ \operatorname{div}\left((1-i \delta) A_{2} \nabla v_{2, \delta}\right)-\lambda_{0} \Sigma_{2} v_{2, \delta}+i \delta v_{2, \delta}=g_{2} & \text { in } \Omega, \\ v_{1, \delta}=v_{2, \delta}, \quad(1+i \delta) A_{1} \nabla v_{1, \delta} \cdot v=(1-i \delta) A_{2} \nabla v_{2, \delta} \cdot v & \text { on } \Gamma .\end{cases}
$$

Moreover,

$$
\left\|v_{\delta}\right\|_{H^{1}(\Omega)}^{2} \leq \frac{C}{\delta}\|g\|_{L^{2}(\Omega)}\left\|v_{\delta}\right\|_{L^{2}(\Omega)},
$$

for some positive constant $C$ independent of $g$ and $\delta$. Consequently,

$$
\left\|v_{\delta}\right\|_{H^{1}(\Omega)} \leq \frac{C}{\delta}\|g\|_{L^{2}(\Omega)} .
$$

Proof Set

$$
X:=\left\{\phi=\left(\phi_{1}, \phi_{2}\right) \in\left[H^{1}(\Omega)\right]^{2}: \phi_{1}-\phi_{2} \in H_{0}^{1}(\Omega)\right\} .
$$

Then $X$ is a Hilbert space equipped the scalar product:

$$
\left\langle\left(\varphi_{1}, \varphi_{2}\right),\left(\phi_{1}, \phi_{2}\right)\right\rangle_{X}:=\left\langle\varphi_{1}, \phi_{1}\right\rangle_{H^{1}(\Omega)}+\left\langle\varphi_{2}, \phi_{2}\right\rangle_{H^{1}(\Omega)} .
$$

Let $a$ be the bilinear form on $X \times X$ defined as follows, for $\varphi=\left(\varphi_{1}, \varphi_{2}\right), \phi=\left(\phi_{1}, \phi_{2}\right) \in X$,

$$
\begin{aligned}
a(\varphi, \phi)= & \int_{\Omega}(1+i \delta)\left\langle A_{1} \nabla \varphi_{1}, \nabla \phi_{1}\right\rangle+\int_{\Omega} \lambda_{0} \Sigma_{1} \varphi_{1} \bar{\phi}_{1}+\int_{\Omega} i \delta \varphi_{1} \bar{\phi}_{1} \\
& -\left[\int_{\Omega}(1-i \delta)\left\langle A_{2} \nabla \varphi_{2}, \nabla \phi_{2}\right\rangle+\lambda_{0} \int_{\Omega} \Sigma_{2} \varphi_{2} \bar{\phi}_{2}-\int_{\Omega} i \delta \varphi_{2} \bar{\phi}_{2}\right],
\end{aligned}
$$

and let $b$ be the linear form on $X$ given by

$$
b(\phi)=\int_{\Omega}-g_{1} \bar{\phi}_{1}+g_{2} \bar{\phi}_{2} .
$$

It is easy to check that $a$ is a continuous on $X \times X$ and $b$ is continuous on $X$; moreover,

$$
|b(\phi)| \leq\left\|\left(g_{1}, g_{2}\right)\right\|_{L^{2}(\Omega)}\left\|\left(\phi_{1}, \phi_{2}\right)\right\|_{L^{2}(\Omega)} .
$$

On the other hand, by considering the imaginary part of $a$, we have

$$
\Im(a(\phi, \phi)) \geq C \delta\|\phi\|_{X}^{2} ;
$$

which implies the coercivity of $a$. By Lax-Milgram's theorem, there exists a unique $\varphi \in X$ such that

$$
a(\varphi, \phi)=b(\phi) \text { for all } \phi \in X .
$$

One can check that $\left(v_{1, \delta}, v_{2, \delta}\right)=\varphi$ is a solution of (2.29); moreover, if $\left(v_{1, \delta}, v_{2, \delta}\right) \in X$ is a solution of (2.29) then $\varphi=\left(v_{1, \delta}, v_{2, \delta}\right)$ is a solution of (2.36). The proof is complete.

Let $\tau>0$ be such that (1.8) holds in $\Omega_{\tau}$. Define

$$
\mathcal{H}(\Omega):=\left\{\left(u_{1}, u_{2}\right) \in\left[H_{l o c}^{1}(\Omega) \cap L^{2}(\Omega)\right]^{2} ; u_{1}-u_{2} \in H_{0}^{1}(\Omega),\left\|\left(u_{1}, u_{2}\right)\right\|_{\mathcal{H}(\Omega)}<+\infty\right\},
$$

where, for $u=\left(u_{1}, u_{2}\right)$ and $v=\left(v_{1}, v_{2}\right)$ in $\left[H_{l o c}^{1}(\Omega)\right]^{2}$,

$$
\langle u, v\rangle_{\mathcal{H}(\Omega)}=\int_{\Omega} \nabla\left(u_{1}-u_{2}\right) \nabla\left(\bar{v}_{1}-\bar{v}_{2}\right)+\int_{\Omega \backslash \Omega_{\tau}} \nabla u \nabla \bar{v}+\int_{\Omega_{\tau}}\left\langle\left[A_{1}-A_{2}\right] \nabla u, \nabla v\right\rangle+\int_{\Omega} u \bar{v} .
$$


One can check that $\mathcal{H}(\Omega)$ equipped the scalar product in (2.38) is a Hilbert space.

We have

Definition 1 Let $\left(g_{1}, g_{2}\right) \in\left[L^{2}(\Omega)\right]^{2}$. A pair of functions $\left(v_{1}, v_{2}\right) \in \mathcal{H}(\Omega)$ is called a weak solution of (2.1) if

$$
\begin{cases}\operatorname{div}\left(A_{1} \nabla v_{1}\right)-\lambda_{0} \Sigma_{1} v_{1}=g_{1} & \text { in } \Omega, \\ \operatorname{div}\left(A_{2} \nabla v_{2}\right)-\lambda_{0} \Sigma_{2} v_{2}=g_{2} & \text { in } \Omega, \\ \left(A_{1} \nabla v_{1}-A_{2} \nabla v_{2}\right) \cdot v=0 & \text { on } \Gamma .\end{cases}
$$

Remark 3 Since $\operatorname{div}\left(A_{1} \nabla v_{1}-A_{2} \nabla v_{2}\right) \in L^{2}(\Omega)$ and $A_{1} \nabla v_{1}-A_{2} \nabla v_{2}=A_{1} \nabla\left(v_{1}-v_{2}\right)+$ $\left(A_{1}-A_{2}\right) \nabla v_{2} \in L^{2}(\Omega)$ by (2.38). The last identity on $\Gamma$ in (2.39) makes sense.

Using Lemmas 1, 5, and 7 one can construct a weak solution $\left(v_{1}, v_{2}\right) \in \mathcal{H}(\Omega)$ of (2.1). More precisely, we have

Proposition 4 Assume (1.8) and let $\delta \in(0,1), g=\left(g_{1}, g_{2}\right) \in\left[L^{2}(\Omega)\right]^{2}$ and $v_{\delta}=$ $\left(v_{1, \delta}, v_{2, \delta}\right) \in\left[H^{1}(\Omega)\right]^{2}$ be the unique solution of (2.29). There exists $\Lambda_{0}>1$ such that if $\lambda_{0}>\Lambda_{0}$ then

$$
\left\|v_{\delta}\right\|_{\mathcal{H}(\Omega)} \leq C\|g\|_{L^{2}(\Omega)},
$$

for some positive constant $C$ independent of $\delta$ and $g$. As a consequence, there exists a weak solution $v=\left(v_{1}, v_{2}\right) \in \mathcal{H}(\Omega)$ of (2.1) such that

$$
\|v\|_{\mathcal{H}(\Omega)} \leq C\|g\|_{L^{2}(\Omega)} .
$$

Proof Applying Lemma 7, we have

$$
\left\|v_{\delta}\right\|_{H^{1}(\Omega)} \leq \frac{C}{\delta}\|g\|_{L^{2}(\Omega)} .
$$

Here and in what follows in the proof, $C$ denotes a positive constant independent of $\delta$ and $g$. Rewriting the system of $v_{\delta}$, we get

$$
\begin{cases}\operatorname{div}\left(A_{1} \nabla v_{1, \delta}\right)-\lambda_{0} \Sigma_{1} v_{1, \delta}=g_{1, \delta} & \text { in } \Omega, \\ \operatorname{div}\left(A_{2} \nabla v_{2, \delta}\right)-\lambda_{0} \Sigma_{2} v_{2, \delta}=g_{2, \delta} & \text { in } \Omega, \\ A_{1} \nabla v_{1, \delta} \cdot v-A_{2} \nabla v_{2, \delta} \cdot v=h_{\delta} & \text { on } \Gamma\end{cases}
$$

where

$$
g_{1, \delta}=\frac{1}{1+i \delta}\left(g_{1}+i \delta v_{1, \delta}-i \delta \lambda_{0} \Sigma_{1} v_{1, \delta}\right), \quad g_{2, \delta}=\frac{1}{1-i \delta}\left(g_{2}-i \delta v_{2, \delta}+i \delta \lambda_{0} \Sigma_{2} v_{2, \delta}\right),
$$

and

$$
h_{\delta}=-i \delta\left(A_{1} \nabla v_{1, \delta}+A_{2} \nabla v_{2, \delta}\right) \cdot v .
$$

Denote $g_{\delta}=\left(g_{1, \delta}, g_{2, \delta}\right)$. For $\lambda_{0} \geq \Lambda_{0}$ and for large $\Lambda_{0}$, we have, by Lemmas 1 and 5 and (2.42),

$$
\left\|v_{\delta}\right\|_{\mathcal{H}(\Omega)}^{2} \leq C\left(\mathcal{M}\left(v_{\delta}, g_{\delta}, h_{\delta}\right)+\left\|g_{\delta}\right\|_{L^{2}(\Omega)}^{2}\right) \leq C\|g\|_{L^{2}(\Omega)}^{2}+C\|g\|_{L^{2}(\Omega)}\|v\|_{L^{2}(\Omega)} .
$$

Here we used the fact that, by (2.42),

$$
\left\|g_{\delta}\right\|_{L^{2}(\Omega)} \leq C\|g\|_{L^{2}(\Omega)}
$$

and in addition the trace theory and (2.30), 


$$
\begin{aligned}
\left\|h_{\delta}\right\|_{H^{-1 / 2}(\Gamma)}\left\|v_{\delta}\right\|_{H^{1 / 2}(\Gamma)} & \leq C \delta\left(\left\|g_{\delta}\right\|_{L^{2}(\Omega)}+\left\|v_{\delta}\right\|_{H^{1}(\Omega)}\right)\left\|v_{\delta}\right\|_{H^{1}(\Omega)} \\
& \leq C\left(\|g\|_{L^{2}(\Omega)}^{2}+\|g\|_{L^{2}(\Omega)}\left\|v_{\delta}\right\|_{L^{2}(\Omega)}\right) .
\end{aligned}
$$

We derive from (2.45) that

$$
\left\|v_{\delta}\right\|_{\mathcal{H}(\Omega)} \leq C\|g\|_{L^{2}(\Omega)} ;
$$

which is (2.40). Assertion (2.41) follows from (2.40) by a standard compactness argument. The details are left to the reader.

We next discuss the uniqueness of the weak solutions. To obtain the uniqueness, one needs to show that $\left(v_{1}, v_{2}\right)=(0,0)$ if $\left(v_{1}, v_{2}\right)$ is a weak solution of (2.39) with $g_{1}=g_{2}=0$. A natural way for this is to obtain (2.6) and (2.7) with $g_{1}=g_{2}=0$ and $h=0$ for $\left(v_{1}, v_{2}\right)$ and then to apply Lemma 5. Note that estimate (2.6) follows from (2.4) and estimate (2.7) follows from (2.5). It is not clear that (2.4) and (2.5) hold for $\left(v_{1}, v_{2}\right) \in \mathcal{H}(\Omega)$ which satisfies (2.39). One way to remedy the situation is to consider only solutions of (2.39) which come from the limiting absorption principle process given in Lemma 7. Even making this restriction, the proof of the uniqueness is still not straightforward since (2.4) and (2.5) are non-linear with respect to $\left(v_{1}, v_{2}\right)$. To overcome this difficulty, we use an kind of relaxation argument which we now introduce. Consider $\left(\hat{v}_{1}, \hat{v}_{2}\right) \in \mathcal{H}(\Omega)$ and $\left(\hat{g}_{1}, \hat{g}_{2}\right) \in\left[L^{2}(\Omega)\right]^{2}$ such that

$$
\begin{cases}\operatorname{div}\left(A_{1} \nabla \hat{v}_{1}\right)-\lambda_{0} \Sigma_{1} \hat{v}_{1}=\hat{g}_{1} & \text { in } \Omega, \\ \operatorname{div}\left(A_{2} \nabla \hat{v}_{2}\right)-\lambda_{0} \Sigma_{2} \hat{v}_{2}=\hat{g}_{2} & \text { in } \Omega, \\ \left(A_{1} \nabla \hat{v}_{1}-A_{2} \nabla \hat{v}_{2}\right) \cdot v=0 & \text { on } \Gamma .\end{cases}
$$

Let $\left(g_{1}, g_{2}\right) \in\left[L^{2}(\Omega)\right]^{2}$ and $\left(v_{1}, v_{2}\right) \in\left[H^{1}(\Omega)\right]^{2}$ be a solution of the system

$$
\begin{cases}\operatorname{div}\left(A_{1} \nabla v_{1}\right)-\lambda_{0} \Sigma_{1} v_{1}=g_{1} & \text { in } \Omega, \\ \operatorname{div}\left(A_{2} \nabla v_{2}\right)-\lambda_{0} \Sigma_{2} v_{2}=g_{2} & \text { in } \Omega, \\ v_{1}=v_{2} & \text { on } \Gamma .\end{cases}
$$

Set $w=v_{1}-v_{2}$ and $\hat{w}=\hat{v}_{1}-\hat{v}_{2}$. We claim that

$$
\begin{aligned}
\int_{\Omega}\left\langle A_{1} \nabla w, \nabla \hat{w}\right\rangle+\lambda_{0} \Sigma_{1} w \overline{\hat{w}}= & -\int_{\Omega}\left(g_{1}-g_{2}\right) \overline{\hat{w}}+\int_{\Omega} \lambda_{0}\left(\Sigma_{2}-\Sigma_{1}\right) v_{2} \overline{\hat{w}} \\
& +\int_{\Omega}\left\langle\left[A_{2}-A_{1}\right] \nabla v_{2}, \nabla \hat{w}\right\rangle
\end{aligned}
$$

and

$$
\begin{aligned}
& \int_{\Omega}\left\langle\left[A_{1}-A_{2}\right] \nabla v_{2}, \nabla \hat{w}\right\rangle+\left\langle\left[A_{1}-A_{2}\right] \nabla v_{2}, \nabla \hat{v}_{2}\right\rangle+\lambda_{0}\left(\Sigma_{1}-\Sigma_{2}\right) v_{2} \overline{\hat{v}}_{2} \\
& \quad=\int_{\Omega} g_{2} \overline{\hat{w}}-\left(\overline{\hat{g}}_{1}-\overline{\hat{g}}_{2}\right) v_{2}+\lambda_{0}\left(\Sigma_{2}-\Sigma_{1}\right) v_{2} \overline{\hat{w}} .
\end{aligned}
$$

Indeed, we have

$$
\operatorname{div}\left(A_{1} \nabla w\right)-\lambda_{0} \Sigma_{1} w=g_{1}-g_{2}-\lambda_{0}\left(\Sigma_{2}-\Sigma_{1}\right) v_{2}+\operatorname{div}\left(\left[A_{2}-A_{1}\right] \nabla v_{2}\right) \text { in } \Omega .
$$

Multiplying this equation by $\overline{\hat{w}}$ and integrating in $\Omega$, we obtain (2.48). On the other hand, multiplying the equation of $v_{2}$ by $\overline{\hat{w}}$ and integrating in $\Omega$, we derive that

$$
\int_{\Omega}-\left\langle A_{2} \nabla v_{2}, \nabla \hat{w}\right\rangle-\lambda_{0} \Sigma_{2} v_{2} \overline{\hat{w}}=\int_{\Omega} g_{2} \overline{\hat{w}} .
$$


We have

$$
-A_{2} \nabla \hat{w}=\left(A_{1}-A_{2}\right) \nabla \hat{w}-A_{1} \nabla \hat{w}+\left(A_{2}-A_{1}\right) \nabla \hat{v}_{2}+\left(A_{1}-A_{2}\right) \nabla \hat{v}_{2} \text { in } \Omega
$$

and, as in (2.12),

$$
\operatorname{div}\left(A_{1} \nabla \hat{w}\right)-\operatorname{div}\left(\left[A_{2}-A_{1}\right] \nabla \hat{v}_{2}\right)=\lambda_{0} \Sigma_{1} \hat{w}+\hat{g}_{1}-\hat{g}_{2}-\lambda_{0}\left(\Sigma_{2}-\Sigma_{1}\right) \hat{v}_{2} \text { in } \Omega .
$$

Since

$$
\left(A_{1} \nabla \hat{w}-\left(A_{2}-A_{1}\right) \nabla \hat{v}_{2}\right) \cdot v=\left(A_{1} \nabla \hat{v}_{1}-A_{2} \nabla \hat{v}_{2}\right) \cdot v=0 \text { on } \Gamma,
$$

it follows from (2.50) that

$$
\begin{aligned}
& \int_{\Omega}\left\langle\left[A_{1}-A_{2}\right] \nabla v_{2}, \nabla \hat{w}\right\rangle+\left\langle\left[A_{1}-A_{2}\right] \nabla v_{2}, \nabla \hat{v}_{2}\right\rangle+\lambda_{0}\left(\Sigma_{1}-\Sigma_{2}\right) v_{2} \overline{\hat{v}}_{2} \\
& \quad=\int_{\Omega} g_{2} \overline{\hat{w}}-\left(\overline{\hat{g}}_{1}-\overline{\hat{g}}_{2}\right) v_{2}+\lambda_{0}\left(\Sigma_{2}-\Sigma_{1}\right) v_{2} \overline{\hat{w}} .
\end{aligned}
$$

which is (2.49).

We are ready to introduce the notion of viscosity solutions of (2.1).

Definition 2 Let $\left(g_{1}, g_{2}\right) \in\left[L^{2}(\Omega)\right]^{2}$. A weak solution $\left(v_{1}, v_{2}\right) \in \mathcal{H}(\Omega)$ of (2.39) is called a viscosity solution if (2.48) and (2.49) hold for any $\left(\hat{v}_{1}, \hat{v}_{2}\right) \in \mathcal{H}(\Omega)$ and $\left(\hat{g}_{1}, \hat{g}_{2}\right) \in\left[L^{2}(\Omega)\right]^{2}$ satisfying (2.47).

We are now in the position to state and prove the crucial result of this section.

Proposition 5 Assume (1.8). There exists $\Lambda_{0}>1$ such that for $\lambda_{0} \geq \Lambda_{0}$ and for $g=$ $\left(g_{1}, g_{2}\right) \in\left[L^{2}(\Omega)\right]^{2}$, there exists a unique viscosity solution $v=\left(v_{1}, v_{2}\right) \in \mathcal{H}(\Omega)$ of $(2.1)$. Moreover,

$$
\|v\|_{\mathcal{H}(\Omega)} \leq C\|g\|_{L^{2}(\Omega)},
$$

for some positive constant $C$ independent of $g$.

Proof Let $v_{\delta}=\left(v_{1, \delta}, v_{2, \delta}\right) \in\left[H^{1}(\Omega)\right]^{2}$ be the unique solution of (2.29) and set $w_{\delta}=$ $v_{1, \delta}-v_{2, \delta}$. By Proposition 4, we have, for large $\Lambda_{0}$,

$$
\left\|v_{\delta}\right\|_{\mathcal{H}(\Omega)} \leq C\|g\|_{L^{2}(\Omega)} .
$$

Let $v$ be the weak limit of $\left(v_{\delta_{n}}\right)$ in $\mathcal{H}(\Omega)$ for some sequence $\left(\delta_{n}\right) \rightarrow 0$ such that $w_{\delta_{n}} \rightarrow w=$ $v_{1}-v_{2}$ weakly in $H^{1}(\Omega)$. Then $v$ is a weak solution of (2.1). We prove that $v$ is a viscosity solution. Define $g_{1, \delta}, g_{2, \delta}$ via (2.44). Then, by (2.48) and (2.49),

$$
\begin{aligned}
\int_{\Omega}\left\langle A_{1} \nabla w_{\delta}, \nabla \hat{w}\right\rangle+\lambda_{0} \Sigma_{1} w_{\delta} \overline{\hat{w}}= & -\int_{\Omega}\left(g_{1, \delta}-g_{2, \delta}\right) \overline{\hat{w}}+\int_{\Omega} \lambda_{0}\left(\Sigma_{2}-\Sigma_{1}\right) v_{2, \delta} \overline{\hat{w}} \\
& +\int_{\Omega}\left\langle\left[A_{2}-A_{1}\right] \nabla v_{2, \delta}, \nabla \hat{w}\right\rangle
\end{aligned}
$$

and

$$
\begin{aligned}
& \int_{\Omega}\left\langle\left[A_{1}-A_{2}\right] \nabla v_{2, \delta}, \nabla \hat{w}\right\rangle+\left\langle\left[A_{1}-A_{2}\right] \nabla v_{2, \delta}, \nabla \hat{v}_{2}\right\rangle+\lambda_{0}\left(\Sigma_{1}-\Sigma_{2}\right) v_{2, \delta} \overline{\hat{v}}_{2} \\
& \quad=\int_{\Omega} g_{2, \delta} \overline{\hat{w}}-\left(\overline{\hat{g}}_{1}-\overline{\hat{g}}_{2}\right) v_{2, \delta}+\lambda_{0}\left(\Sigma_{2}-\Sigma_{1}\right) v_{2, \delta} \overline{\hat{w}} .
\end{aligned}
$$


By (2.52), it follows from (2.30) of Lemma 7 that

$$
\left\|v_{\delta}\right\|_{H^{1}(\Omega)} \leq \frac{C}{\sqrt{\delta}}\|g\|_{L^{2}(\Omega)} .
$$

By letting $\delta \rightarrow 0$ in (2.53) and (2.54), and using (2.55), one obtains (2.48) and (2.49) for $v$. Hence $v$ is a viscosity solution. The uniqueness of $v$ follows from the definition of viscosity solutions as follows. Assume that $\left(v_{1}, v_{2}\right)$ and $\left(\widetilde{v}_{1}, \widetilde{v}_{2}\right)$ are two viscosity solutions. Set

$$
\left(V_{1}, V_{2}\right)=\left(v_{1}-\widetilde{v}_{1}, v_{2}-\widetilde{v}_{2}\right) \text { in } \Omega .
$$

Then $\left(V_{1}, V_{2}\right)$ is a viscosity solution corresponding to the pair of data $(0,0)$. By choosing $\left(\hat{v}_{1}, \hat{v}_{2}\right)=\left(V_{1}, V_{2}\right)$, one obtains (2.4) and (2.5) where $\left(v_{1}, v_{2}\right)=\left(V_{1}, V_{2}\right)$ and $\left(g_{1}, g_{2}, h\right)=$ $(0,0,0)$. It follows from Lemma 1 that (2.6) and (2.7) holds with $\left(g_{1}, g_{2}, h\right)=(0,0,0)$. This implies $\left(V_{1}, V_{2}\right)=(0,0)$ by Lemma 5 for large $\lambda_{0}$. The proof is complete.

Fix $\lambda_{0}>\Lambda_{0}$ where $\Lambda_{0}$ is the constant in Proposition 5. Define

$$
\begin{aligned}
T_{1}:\left[L^{2}(\Omega)\right]^{2} & \rightarrow\left[L^{2}(\Omega)\right]^{2} \\
\left(f_{1}, f_{2}\right) & \mapsto\left(u_{1}, u_{2}\right),
\end{aligned}
$$

where $\left(u_{1}, u_{2}\right) \in \mathcal{H}(\Omega)$ is the unique viscosity solution of (2.1) with $\left(g_{1}, g_{2}\right)=$ $\left(\Sigma_{1} f_{1}, \Sigma_{2} f_{2}\right)$. Using (2.51) and applying Lemma 3, we derive that $T_{1}$ is compact.

We are ready to give the

Proof of Theorem 1 By the theory of compact operator see, e.g., [4], the spectrum of $T_{1}$ is discrete. It is clear that if $\left(u_{1}, u_{2}\right)$ is an eigenfunction pair of the ITE problem corresponding to the eigenvalue $\lambda$ then $\left(u_{1}, u_{2}\right)$ is eigenfunction pair of $T_{1}$ corresponding to the eigenvalue $\frac{1}{\lambda-\lambda_{0}}$. Hence, the spectrum of the ITE problem is discrete.

Remark 4 The condition $0 \leq \alpha<2$ is necessary to guarantee the compactness of $T_{1}$.

Remark 5 Theorem 1 also holds if the condition $\Sigma_{1}-\Sigma_{2} \geq 0$ in (1.8) is replaced by $\Sigma_{1}-\Sigma_{2} \geq-\hat{c}$ for some small positive constant $\hat{c}$ since Lemma 5 holds in this case (see Remark 1).

\section{On the case $A_{1} \geq A_{2}$ and $\Sigma_{1}$ not greater than $\Sigma_{2}$ in a neighborhood of $\Gamma$}

This section contains two sections. In the first one, we deal with the case $A_{1} \geq A_{2}$ and $\Sigma_{1} \leq \Sigma_{2}$ in a neighborhood of $\Gamma$ and give the proof of Theorem 2. In the second one, we deal with the case $A_{1} \geq A_{2}$ globally in $\Omega$; the main result in this section is Proposition 8 .

\subsection{On the case $A_{1} \geq A_{2}$ and $\Sigma_{1} \leq \Sigma_{2}$ in a neighborhood of $\Gamma$-Proof of Theorem 2}

The section is devoted to the proof of Theorem 2. As mentioned in the introduction, we first establish the well-posedness of the following system, for some $\lambda_{0}>0$ (small),

$$
\begin{cases}\operatorname{div}\left(A_{1} \nabla u_{1}\right)-i \lambda_{0} \Sigma_{1} u_{1}=g_{1} & \text { in } \Omega, \\ \operatorname{div}\left(A_{2} \nabla u_{2}\right)-i \lambda_{0} \Sigma_{2} u_{2}=g_{2} & \text { in } \Omega, \\ u_{1}=u_{2}, \quad A_{1} \nabla u_{1} \cdot v=A_{2} \nabla u_{2} \cdot v & \text { on } \Gamma,\end{cases}
$$

for a given pair $\left(g_{1}, g_{2}\right)$ in an appropriate space. We then define the operator 
$T_{2}\left(f_{1}, f_{2}\right)=\left(u_{1}, u_{2}\right)$ where $\left(u_{1}, u_{2}\right)$ is the unique solution of (3.1)

$$
\text { with }\left(g_{1}, g_{2}\right)=\left(\Sigma_{1} f_{1}, \Sigma_{2} f_{2}\right)
$$

and prove the compactness for $T_{2}$. System (3.1) is slightly different from the one (2.1) where the constant $i$ appears in front of $\lambda_{0}$. This modification is necessary for the proof of the uniqueness. As in the proof of Theorem 1, similar a priori estimates hold for (3.1) (Lemma 8). Nevertheless, the uniqueness of (3.1) even for smooth solutions does not follow directly from the a priori estimates as in the proof of Theorem 1. Additional arguments are required and the condition on the largeness of $\Sigma_{2}$ is involved (Lemma 10). Beside this point, the proof of Theorem 2 is in the spirit of the one of Theorem 1.

We now process the proof of Theorem 2. We first establish a variant of Lemma 1.

Lemma 8 Let $\lambda_{0}>0, g=\left(g_{1}, g_{2}\right) \in\left[L^{2}(\Omega)\right]^{2}$, and $h \in H^{-1 / 2}(\Gamma)$. Assume that $v=$ $\left(v_{1}, v_{2}\right) \in\left[H^{1}(\Omega)\right]^{2}$ satisfy the system

$$
\begin{cases}\operatorname{div}\left(A_{1} \nabla v_{1}\right)-i \lambda_{0} \Sigma_{1} v_{1}=g_{1} & \text { in } \Omega \\ \operatorname{div}\left(A_{2} \nabla v_{2}\right)-i \lambda_{0} \Sigma_{2} v_{2}=g_{2} & \text { in } \Omega \\ v_{1}=v_{2}, \quad A_{1} \nabla v_{1} \cdot v=A_{2} \nabla v_{2} \cdot v+h & \text { on } \Gamma\end{cases}
$$

We have, with $w=v_{2}-v_{1}$,

$$
\begin{aligned}
\int_{\Omega}\left\langle A_{1} \nabla w, \nabla w\right\rangle+i \lambda_{0} \Sigma_{1}|w|^{2}= & -\int_{\Omega}\left(g_{1}-g_{2}\right) \bar{w}+\int_{\Omega} i \lambda_{0}\left(\Sigma_{2}-\Sigma_{1}\right) v_{2} \bar{w} \\
& +\int_{\Omega}\left\langle\left[A_{2}-A_{1}\right] \nabla v_{2}, \nabla w\right\rangle
\end{aligned}
$$

and

$$
\begin{aligned}
& \int_{\Omega}\left\langle\left[A_{1}-A_{2}\right] \nabla v_{2}, \nabla w\right\rangle+\left\langle\left[A_{1}-A_{2}\right] \nabla v_{2}, \nabla v_{2}\right\rangle+i \lambda_{0}\left(\Sigma_{2}-\Sigma_{1}\right)\left|v_{2}\right|^{2} \\
& \quad=\int_{\Omega} g_{2} \bar{w}-\left(\bar{g}_{1}-\bar{g}_{2}\right) v_{2}+i \lambda_{0}\left(\Sigma_{1}+\Sigma_{2}\right) v_{2} \bar{w}+\int_{\Gamma} \bar{h} v_{2} .
\end{aligned}
$$

As a consequence of (3.4) and (3.5), we obtain

$$
\left.\left.\lambda_{0}\left|\int_{\Omega}\left(\Sigma_{2}-\Sigma_{1}\right)\right| v_{2}\right|^{2}\left|\leq 4 \mathcal{M}(v, g, h)+\lambda_{0} \int_{\Omega} \Sigma_{1}\right| w\right|^{2}+2 \lambda_{0} \int_{\Omega} \Sigma_{1}\left|v_{2}\right||w|
$$

and

$$
\begin{aligned}
& \int_{\Omega}\left\langle A_{1} \nabla w, \nabla w\right\rangle+\int_{\Omega}\left\langle\left[A_{1}-A_{2}\right] \nabla v_{2}, \nabla v_{2}\right\rangle \\
& \quad \leq 4 \mathcal{M}(v, g, h)+2 \int_{\Omega}\left|\left\langle\left[A_{2}-A_{1}\right] \nabla v_{2}, \nabla w\right\rangle\right|+2 \lambda_{0} \int_{\Omega} \Sigma_{2}\left|v_{2}\right||w|,
\end{aligned}
$$

where $\mathcal{M}(v, g, h)$ is defined in (2.8).

Proof The proof is in the spirit of the one of Lemma 1; nevertheless, different coefficients appear in the conclusion due to the effect of the constant $i$ in the front of $\lambda_{0}$. For the convenient of the reader, we present the proof. From the definition of $w$, we derive that $w=0$ on $\Gamma$ and

$$
\operatorname{div}\left(A_{1} \nabla w\right)-i \lambda_{0} \Sigma_{1} w=g_{1}-g_{2}-i \lambda_{0}\left(\Sigma_{2}-\Sigma_{1}\right) v_{2}+\operatorname{div}\left(\left[A_{2}-A_{1}\right] \nabla v_{2}\right) \text { in } \Omega .
$$

Multiplying this equation by $\bar{w}$ and integrating on $\Omega$, we have 


$$
\begin{aligned}
\int_{\Omega}\left\langle A_{1} \nabla w, \nabla w\right\rangle+i \lambda_{0} \Sigma_{1}|w|^{2}= & -\int_{\Omega}\left(g_{1}-g_{2}\right) \bar{w}+\int_{\Omega} i \lambda_{0}\left(\Sigma_{2}-\Sigma_{1}\right) v_{2} \bar{w} \\
& +\int_{\Omega}\left\langle\left[A_{2}-A_{1}\right] \nabla v_{2}, \nabla w\right\rangle ;
\end{aligned}
$$

which is (3.4). Multiplying the equation of $v_{2}$ by $\bar{w}$ and integrating on $\Omega$, we obtain

$$
\int_{\Omega}-\left\langle A_{2} \nabla v_{2}, \nabla w\right\rangle-i \lambda_{0} \Sigma_{2} v_{2} \bar{w}=\int_{\Omega} g_{2} \bar{w}
$$

It is clear that

$$
-A_{2} \nabla w=\left(A_{1}-A_{2}\right) \nabla w-A_{1} \nabla w+\left(A_{2}-A_{1}\right) \nabla v_{2}+\left(A_{1}-A_{2}\right) \nabla v_{2} \text { in } \Omega,
$$

and, by (3.8),

$$
\operatorname{div}\left(A_{1} \nabla w\right)-\operatorname{div}\left(\left[A_{2}-A_{1}\right] \nabla v_{2}\right)=i \lambda_{0} \Sigma_{1} w+g_{1}-g_{2}-i \lambda_{0}\left(\Sigma_{2}-\Sigma_{1}\right) v_{2} \text { in } \Omega .
$$

Since

$$
\left(A_{1} \nabla w-\left(A_{2}-A_{1}\right) \nabla v_{2}\right) \cdot v=\left(A_{1} \nabla v_{1}-A_{2} \nabla v_{2}\right) \cdot v=h \text { on } \Gamma,
$$

it follows from (3.10), (3.11), and (3.12) that

$$
\begin{aligned}
& \int_{\Omega}\left\langle\left[A_{1}-A_{2}\right] \nabla v_{2}, \nabla w\right\rangle+\left\langle\left[A_{1}-A_{2}\right] \nabla v_{2}, \nabla v_{2}\right\rangle+i \lambda_{0}\left(\Sigma_{2}-\Sigma_{1}\right)\left|v_{2}\right|^{2} \\
& =\int_{\Omega} g_{2} \bar{w}-\left(\bar{g}_{1}-\bar{g}_{2}\right) v_{2}+i \lambda_{0}\left(\Sigma_{1}+\Sigma_{2}\right) v_{2} \bar{w}+\int_{\Gamma} \bar{h} v_{2} ;
\end{aligned}
$$

which is (3.5).

Subtracting (3.4) from (3.13) and considering the imaginary part yields

$$
\left.\left.\lambda_{0}\left|\int_{\Omega}\left(\Sigma_{2}-\Sigma_{1}\right)\right| v_{2}\right|^{2}\left|\leq 4 \mathcal{M}(v, g, h)+\lambda_{0} \int_{\Omega} \Sigma_{1}\right| w\right|^{2}+2 \lambda_{0} \int_{\Omega} \Sigma_{1}\left|v_{2}\right||w| ;
$$

which is (3.6). Adding (3.4) and (3.13) and considering the real part implies

$$
\begin{aligned}
& \int_{\Omega}\left\langle A_{1} \nabla w, \nabla w\right\rangle+\int_{\Omega}\left\langle\left[A_{1}-A_{2}\right] \nabla v_{2}, \nabla v_{2}\right\rangle \\
& \quad \leq 4 \mathcal{M}(v, g, h)+2 \lambda_{0} \int_{\Omega} \Sigma_{2}\left|v_{2}\right||w|+2\left|\int_{\Omega}\left\langle\left[A_{2}-A_{1}\right] \nabla v_{2}, \nabla w\right\rangle\right| .
\end{aligned}
$$

which is (3.7). The proof is complete.

Here is a variant of Lemma 2.

Lemma 9 Let $\lambda>1, f \in L^{2}(\Omega)$, A be a matrix-valued function, and $\Sigma$ be real function defined in $\Omega$ such that

$$
\Lambda^{-1} I \leq A \leq \Lambda I \quad \text { and } \quad \Lambda^{-1} \leq \Sigma \leq \Lambda \text { in } \Omega .
$$

Let $u \in H_{\text {loc }}^{1}(\Omega)$ be a solution to the equation $\operatorname{div}(A \nabla u)-i \lambda \Sigma u=f$ in $\Omega$. For all $s>0$, there exist two positive constants $c_{1}$ and $c_{2}$, depending only on $\Lambda, s$, and $\Omega$, such that

$$
\|u\|_{H^{1}\left(\Omega \backslash \Omega_{s}\right)} \leq c_{1} \exp \left(-c_{2} \sqrt{\lambda}\right)\|u\|_{L^{2}\left(\Omega_{s}\right)}+c_{1}\|f\|_{L^{2}(\Omega)} .
$$

Proof The proof of Lemma 9 is similar to the one of Lemma 2. The details are omitted. 
The following result is a variant of Lemma 5 and plays an important role in the proof of the uniqueness in Theorem 2.

Lemma 10 Assume that for some $c, \tau>0$ and for some $0 \leq \alpha<2$,

$$
A_{1}-A_{2} \geq c d_{\Gamma}^{\alpha} I \text { in } \Omega_{\tau} .
$$

Let $\Lambda_{1} \geq 1$ be such that,

$$
\Lambda_{1}^{-1} I \leq A_{j} \leq \Lambda_{1} I \text { for } j=1,2, \quad \text { and } \quad \Lambda_{1}^{-1} \leq \Sigma_{1} \leq \Lambda_{1} \text { in } \Omega .
$$

For every $0<\Lambda_{2}<1$ there exists $K_{1}>1$ depending only on $\Lambda_{1}, \Lambda_{2}, c, \alpha$, and $\tau$ such that if

$$
\Lambda_{2} K \leq \Sigma_{2} \leq K \text { in } \Omega \text {, }
$$

for some $K>K_{1}$, then there exists $\lambda_{0}>0$, depending on $K$, such that if $g=\left(g_{1}, g_{2}\right) \in$ $\left[L^{2}(\Omega)\right]^{2}, h \in H^{-1 / 2}(\Gamma)$, and $v=\left(v_{1}, v_{2}\right) \in \mathcal{H}(\Omega)$ verify

$$
\left\{\begin{array}{l}
\operatorname{div}\left(A_{1} \nabla v_{1}\right)-i \lambda_{0} \Sigma_{1} v_{1}=g_{1} \text { in } \Omega, \\
\operatorname{div}\left(A_{2} \nabla v_{2}\right)-i \lambda_{0} \Sigma_{2} v_{2}=g_{2} \text { in } \Omega,
\end{array}\right.
$$

and (3.6) and (3.7), we have

$$
\|v\|_{\mathcal{H}(\Omega)}^{2} \leq C\left(\mathcal{M}(v, g, h)+\|g\|_{L^{2}(\Omega)}^{2}\right),
$$

for some positive constant $C$ independent of $v, g$, and $h$ where $\mathcal{M}=\mathcal{M}(v, g, h)$ is defined in (2.8). In particular if $v \in\left[H^{1}(\Omega)\right]^{2}$ is a solution of (3.3) then (3.20) holds.

Proof Fix $0<\varepsilon<1$ a small constant which is defined later and set $\lambda_{0}=\varepsilon K^{-1 / 2}$. Note that $\lambda_{0} K=\varepsilon K^{1 / 2}$ is large if $K$ is large; this fact is assumed from now on. We derive from (3.6) and Lemma 9 that, for large $K$,

$$
\int_{\Omega} \lambda_{0} K\left|v_{2}\right|^{2} \leq C \int_{\Omega} \lambda_{0}\left|v_{2}\right||w|+\lambda_{0}|w|^{2}+C \mathcal{M}(v, g, h) .
$$

Here and in what follows in the proof, $C$ denotes a positive constant depending only on $\Lambda_{1}, \Lambda_{2}, \Omega$, and $\tau$; it is independent of $K$ and $\varepsilon$. This implies, for large $K$,

$$
\int_{\Omega} \lambda_{0} K\left|v_{2}\right|^{2} \leq C \int_{\Omega} \lambda_{0}|w|^{2}+C \mathcal{M}(v, g, h) .
$$

Using the fact, for $\gamma>0$,

$\gamma \int_{\Omega_{\tau}}\left\langle\left[A_{1}-A_{2}\right] \nabla w, \nabla w\right\rangle+\frac{1}{\gamma} \int_{\Omega_{\tau}}\left\langle\left[A_{1}-A_{2}\right] \nabla v_{2}, \nabla v_{2}\right\rangle \geq 2 \int_{\Omega_{\tau}}\left|\left\langle\left[A_{1}-A_{2}\right] \nabla w, \nabla v_{2}\right\rangle\right|$ and taking $\gamma>1$ and close to 1 , we derive from (3.7) that

$$
\begin{aligned}
\int_{\Omega}\left\langle A_{1} \nabla w, \nabla w\right\rangle+\int_{\Omega_{\tau}}\left\langle\left[A_{1}-A_{2}\right] \nabla v_{2}, \nabla v_{2}\right\rangle \leq & C \int_{\Omega} \lambda_{0} K\left|v_{2}\right||w|+C \int_{\Omega \backslash \Omega_{\tau}}\left|\nabla v_{2}\right|^{2} \\
& +C \mathcal{M}(v, g, h) .
\end{aligned}
$$

Since

$$
2 \lambda_{0} K\left|v_{2}\right||w| \leq \lambda_{0} K^{3 / 2}\left|v_{2}\right|^{2}+\lambda_{0} K^{1 / 2}|w|^{2},
$$


it follows from (3.21) and (3.22) that

$$
\begin{aligned}
& \int_{\Omega}\left\langle A_{1} \nabla w, \nabla w\right\rangle+\int_{\Omega_{\tau}}\left\langle\left[A_{1}-A_{2}\right] \nabla v_{2}, \nabla v_{2}\right\rangle \\
& \quad \leq C \int_{\Omega} \lambda_{0} K^{1 / 2}|w|^{2}+C \int_{\Omega \backslash \Omega_{\tau}}\left|\nabla v_{2}\right|^{2}+C K^{1 / 2} \mathcal{M}(v, g, h) .
\end{aligned}
$$

Choosing $\varepsilon$ small enough, one can absorb the first term in the RHS of (3.23) by the first term of the LHS of (3.23) (recall that $\lambda_{0} K^{1 / 2}=\varepsilon$ ) and obtains

$$
\int_{\Omega}\left\langle A_{1} \nabla w, \nabla w\right\rangle+\int_{\Omega_{\tau}}\left\langle\left[A_{1}-A_{2}\right] \nabla v_{2}, \nabla v_{2}\right\rangle \leq C \int_{\Omega \backslash \Omega_{\tau}}\left|\nabla v_{2}\right|^{2}+C K^{1 / 2} \mathcal{M}(v, g, h) .
$$

Similar to (2.26) (see Remark 2), we have

$$
\int_{\Omega}\left|v_{2}\right|^{2} \leq C \int_{\Omega} d_{\Gamma}^{\alpha}\left|\nabla v_{2}\right|^{2}+C\left\|g_{2}\right\|_{L^{2}(\Omega)}^{2} .
$$

Estimate (3.20) now follows from (3.24) and (3.25) by applying Lemma 9 with $v=v_{2}, A=$ $A_{2}, \Sigma=\Sigma_{2} / K$, and $\lambda=\lambda_{0} K=\varepsilon K^{1 / 2}$.

The following result is a variant of Lemma 7 and its proof uses essentially Lemma 10.

Lemma 11 Assume that for some $c, \tau>0$ and for some $0 \leq \alpha<2$,

$$
A_{1}-A_{2} \geq c d_{\Gamma}^{\alpha} I \text { in } \Omega_{\tau} .
$$

Let $\Lambda_{1} \geq 1$ be such that

$$
\Lambda_{1}^{-1} I \leq A_{j} \leq \Lambda_{1} I \text { for } j=1,2, \quad \text { and } \quad \Lambda_{1}^{-1} \leq \Sigma_{1} \leq \Lambda_{1} \text { in } \Omega .
$$

For every $0<\Lambda_{2}<1$ there exists $K_{1}>1$ depending only on $\Lambda_{1}, \Lambda_{2}, c, \alpha$, and $\tau$ such that if

$$
\Lambda_{2} K \leq \Sigma_{2} \leq K \text { in } \Omega,
$$

for some $K>K_{1}$, then there exists $\lambda_{0}>0$, depending on $K$, such that for $\delta \in(0,1)$, $g=\left(g_{1}, g_{2}\right) \in\left[L^{2}(\Omega)\right]^{2}$, there exists a unique solution $v_{\delta}=\left(v_{1, \delta}, v_{2, \delta}\right) \in\left[H^{1}(\Omega)\right]^{2}$ of the system

$$
\begin{cases}\operatorname{div}\left((1+\delta) A_{1} \nabla v_{1, \delta}\right)-i \lambda_{0} \Sigma_{1} v_{1, \delta}=g_{1} & \text { in } \Omega, \\ \operatorname{div}\left(A_{2} \nabla v_{2, \delta}\right)-i \lambda_{0} \Sigma_{2} v_{2, \delta}=g_{2} & \text { in } \Omega, \\ v_{1, \delta}=v_{2, \delta}, \quad(1+\delta) A_{1} \nabla v_{1, \delta} \cdot v=A_{2} \nabla v_{2, \delta} \cdot v & \text { on } \Gamma\end{cases}
$$

Moreover,

$$
\left\|v_{\delta}\right\|_{H^{1}(\Omega)}^{2} \leq \frac{C}{\delta}\left(\|g\|_{L^{2}(\Omega)}\left\|v_{\delta}\right\|_{L^{2}(\Omega)}+\|g\|_{L^{2}(\Omega)}^{2}\right),
$$

for some positive constant $C$ independent of $g$ and $\delta$. Consequently,

$$
\left\|v_{\delta}\right\|_{H^{1}(\Omega)} \leq \frac{C}{\delta}\|g\|_{L^{2}(\Omega)} .
$$

Proof Applying Lemma 10 with $A_{1}=(1+\delta) A_{1}$ and $A_{2}=A_{2}$, there exists $K_{1}>1$ depending only on $\Lambda_{1}, \Lambda_{2}, c, \alpha$, and $\tau$ such that if

$$
\Lambda_{2} K \leq \Sigma_{2} \leq K \text { in } \Omega \text {, }
$$


for some $K>K_{1}$, there exists $\lambda_{0}>0$, depending on $K$, such that if $v_{\delta}=\left(v_{1, \delta}, v_{2, \delta}\right) \in$ $\left[H^{1}(\Omega)\right]^{2}(0<\delta<1)$ is a solution of (3.29) then

$$
\begin{aligned}
& \left\|v_{\delta}\right\|_{H^{1}\left(\Omega \backslash \Omega_{\tau / 2}\right)}^{2}+\left\|v_{1, \delta}-v_{2, \delta}\right\|_{H^{1}(\Omega)}^{2}+\left\|v_{\delta}\right\|_{L^{2}(\Omega)}^{2}+\int_{\Omega_{\tau}}\left\langle\left[(1+\delta) A_{1}-A_{2}\right] \nabla v_{\delta}, \nabla v_{\delta}\right\rangle \\
& \leq C \mathcal{M}\left(v_{\delta}, g, 0\right)+C\|g\|_{L^{2}(\Omega)}^{2},
\end{aligned}
$$

for some positive constant $C$ independent of $g, v_{\delta}$, and $\delta$. This implies the uniqueness of $v_{\delta}$ and estimate (3.30). The existence of $v_{\delta}$ follows from Fredholm's theory and can be proceeded as follows. Define

$$
\begin{aligned}
T_{1, \varepsilon}:\left[L^{2}(\Omega)\right]^{2} & \rightarrow\left[L^{2}(\Omega)\right]^{2} \\
\left(g_{1}, g_{2}\right) & \mapsto\left(v_{1}, v_{2}\right),
\end{aligned}
$$

where $\left(v_{1}, v_{2}\right) \in\left[H^{1}(\Omega)\right]^{2}$ is the unique solution of $(2.1)$ where $\left(A_{1}, A_{2}\right)$ and $\left(\Sigma_{1}, \Sigma_{2}\right)$ are replaced by $\left((1+\delta) A_{1}, A_{2}\right)$ and $\left(2 \varepsilon^{-1}, \varepsilon^{-1}\right)$ for small $\varepsilon . T_{1, \varepsilon}$ is well-defined since $T_{1}$ given in (2.56) is well-defined ( $\alpha=0$ in this case). The existence of $v_{\delta}$ follows from the uniqueness of $v_{\delta}$ by applying the Fredholm theory for the operator $I-T_{1, \varepsilon} \circ B:\left[L^{2}(\Omega)\right]^{2} \rightarrow\left[L^{2}(\Omega)\right]^{2}$ where $B:\left[L^{2}(\Omega)\right]^{2} \rightarrow\left[L^{2}(\Omega)\right]^{2}$ is defined by

$$
B\left(v_{1}, v_{2}\right)=\left(i \lambda_{0} \Sigma_{1} v_{1}-2 \varepsilon^{-1} v_{1}, i \lambda_{0} \Sigma_{2} v_{2}-\varepsilon^{-1} v_{2}\right) .
$$

Clearly $B$ is invertible if $\varepsilon$ is small enough. Note that $I-T_{1, \varepsilon} \circ B$ is injective by the uniqueness of (3.29). By the Fredholm theory it is bijective. Hence for any $g \in\left[L^{2}(\Omega)\right]^{2}$ there exists $u \in\left[L^{2}(\Omega)\right]^{2}$ such that $u-T_{1, \varepsilon} \circ B(u)=B^{-1} g$. Set $v=u-B^{-1} g$. Then $T_{1, \varepsilon} \circ B\left(v+B^{-1} g\right)=v$. In other words, $v$ is a solution of (3.29). The proof is complete.

We are now in the position to give the definition of a weak solution of (2.1). Let $\tau>0$ be such that (1.9) holds in $\Omega_{\tau}$. Define $\mathcal{H}(\Omega)$ as in (2.37) with the scalar product given in (2.38). The notion of weak solution is similar to the one in Definition 1 and is given in

Definition 3 Let $\left(g_{1}, g_{2}\right) \in\left[L^{2}(\Omega)\right]^{2}$. A pair of functions $\left(v_{1}, v_{2}\right) \in \mathcal{H}(\Omega)$ is called a weak solution of (2.1) if

$$
\begin{cases}\operatorname{div}\left(A_{1} \nabla v_{1}\right)-i \lambda_{0} \Sigma_{1} v_{1}=g_{1} & \text { in } \Omega, \\ \operatorname{div}\left(A_{2} \nabla v_{2}\right)-i \lambda_{0} \Sigma_{2} v_{2}=g_{2} & \text { in } \Omega, \\ \left(A_{1} \nabla v_{1}-A_{2} \nabla v_{2}\right) \cdot v=0 & \text { on } \Gamma\end{cases}
$$

Using Lemmas 10 and 11, one can construct a weak solution $\left(v_{1}, v_{2}\right) \in \mathcal{H}(\Omega)$ of (3.1). More precisely, we have

Proposition 6 Let $\delta \in(0,1), g=\left(g_{1}, g_{2}\right) \in\left[L^{2}(\Omega)\right]^{2}$, and $v_{\delta}=\left(v_{1, \delta}, v_{2, \delta}\right) \in\left[H^{1}(\Omega)\right]^{2}$ be the unique solution of (3.29). Assume (3.26), (3.27), and (3.28). Let $K>K_{1}>1$ and $\lambda_{0}>0$ be as in Lemma 11. We have

$$
\left\|v_{\delta}\right\|_{\mathcal{H}(\Omega)} \leq C\|g\|_{L^{2}(\Omega)},
$$

for some positive constant $C$ independent of $\delta$ and $\left(g_{1}, g_{2}\right)$. As a consequence, there exists a weak solution $v=\left(v_{1}, v_{2}\right) \in \mathcal{H}(\Omega)$ of (3.1) such that

$$
\|v\|_{\mathcal{H}(\Omega)} \leq C\|g\|_{L^{2}(\Omega)} .
$$

Proof The proof is similar to the one of Proposition 4. However, instead of using Lemmas 1, 5, and 7, one applies Lemmas 8, 10, and 11. The details are left to the reader. 
As in the proof of Theorem 1 in Sect. 2, we introduce the concept of viscosity solutions using a relaxation argument. Let $\left(\hat{v}_{1}, \hat{v}_{2}\right) \in \mathcal{H}(\Omega)$ and $\left(\hat{g}_{1}, \hat{g}_{2}\right) \in\left[L^{2}(\Omega)\right]^{2}$ be such that

$$
\begin{cases}\operatorname{div}\left(A_{1} \nabla \hat{v}_{1}\right)-i \lambda_{0} \Sigma_{1} \hat{v}_{1}=\hat{g}_{1} & \text { in } \Omega, \\ \operatorname{div}\left(A_{2} \nabla \hat{v}_{2}\right)-i \lambda_{0} \Sigma_{2} \hat{v}_{2}=\hat{g}_{2} & \text { in } \Omega, \\ \left(A_{1} \nabla \hat{v}_{1}-A_{2} \nabla \hat{v}_{2}\right) \cdot v & \text { on } \Gamma .\end{cases}
$$

Let $\left(g_{1}, g_{2}\right) \in\left[L^{2}(\Omega)\right]^{2}$ and $\left(v_{1}, v_{2}\right) \in\left[H^{1}(\Omega)\right]^{2}$ be a weak solution of the system

$$
\begin{cases}\operatorname{div}\left(A_{1} \nabla v_{1}\right)-\lambda_{0} \Sigma_{1} v_{1}=g_{1} & \text { in } \Omega, \\ \operatorname{div}\left(A_{2} \nabla v_{2}\right)-\lambda_{0} \Sigma_{2} v_{2}=g_{2} & \text { in } \Omega, \\ v_{1}=v_{2} & \text { on } \Gamma .\end{cases}
$$

Set $w=v_{1}-v_{2}$ and $\hat{w}=\hat{v}_{1}-\hat{v}_{2}$. Involving the same arguments used to derive (2.48) and (2.49), we have

$$
\begin{aligned}
\int_{\Omega}\left\langle A_{1} \nabla w, \nabla \hat{w}\right\rangle+i \lambda_{0} \Sigma_{1} w \overline{\hat{w}}= & -\int_{\Omega}\left(g_{1}-g_{2}\right) \overline{\hat{w}}+\int_{\Omega} i \lambda_{0}\left(\Sigma_{2}-\Sigma_{1}\right) v_{2} \overline{\hat{w}} \\
& +\int_{\Omega}\left\langle\left[A_{2}-A_{1}\right] \nabla v_{2}, \nabla \hat{w}\right\rangle
\end{aligned}
$$

and

$$
\begin{aligned}
& \int_{\Omega}\left\langle\left(A_{1}-A_{2}\right) \nabla v_{2}, \nabla \hat{w}\right\rangle+\left\langle\left(A_{1}-A_{2}\right) \nabla v_{2}, \nabla \hat{v}_{2}\right\rangle+i \lambda_{0}\left(\Sigma_{2}-\Sigma_{1}\right) v_{2} \overline{\hat{v}}_{2} \\
& \quad=\int_{\Omega} g_{2} \overline{\hat{w}}-\left(\overline{\hat{g}}_{1}-\overline{\hat{g}}_{2}\right) v_{2}+i \lambda_{0}\left(\Sigma_{1}+\Sigma_{2}\right) v_{2} \overline{\hat{w}} .
\end{aligned}
$$

We are ready now to define the notion of viscosity solutions of (3.1).

Definition 4 Let $\left(g_{1}, g_{2}\right) \in\left[L^{2}(\Omega)\right]^{2}$. A weak solution $\left(v_{1}, v_{2}\right) \in \mathcal{H}(\Omega)$ of $(3.1)$ is called a viscosity solution if (3.38) and (3.39) hold for any $\left(\hat{v}_{1}, \hat{v}_{2}\right) \in \mathcal{H}(\Omega)$ and $\left(\hat{g}_{1}, \hat{g}_{2}\right) \in\left[L^{2}(\Omega)\right]^{2}$ which satisfy (3.37).

Applying Lemma 8 and using Lemma 11, we can prove

Proposition 7 Assume (3.26), (3.27), and (3.28). Let $K_{1}>1$ and $\lambda_{0}>1$ be as in Lemma 11. For $g=\left(g_{1}, g_{2}\right) \in\left[L^{2}(\Omega)\right]^{2}$, there exists a unique viscosity solution $v=\left(v_{1}, v_{2}\right) \in \mathcal{H}(\Omega)$ of (3.1). Moreover,

$$
\|v\|_{\mathcal{H}(\Omega)} \leq C\|g\|_{L^{2}(\Omega)},
$$

for some positive constant $C$ independent of $\left(g_{1}, g_{2}\right)$.

Proof The proof of Proposition 7 is similar to the one of Proposition 5. The details are left to the reader.

Fix $\lambda_{0}$ in Proposition 7. Define

$$
\begin{aligned}
T_{2}:\left[L^{2}(\Omega)\right]^{2} & \rightarrow\left[L^{2}(\Omega)\right]^{2} \\
\left(f_{1}, f_{2}\right) & \mapsto\left(u_{1}, u_{2}\right),
\end{aligned}
$$

where $\left(u_{1}, u_{2}\right) \in \mathcal{H}(\Omega)$ is the unique viscosity solution of (3.1). The compactness of $T_{2}$ is a consequence of Lemma 3.

We are ready to give 
Proof of Theorem 2 By the theory of compact operator, the spectrum of $T_{2}$ is discrete. It is clear that if $\left(u_{1}, u_{2}\right)$ is an eigenfunction pair of the ITE problem corresponding to $\lambda$ then $\left(u_{1}, u_{2}\right)$ is eigenfunction pair of $T$ corresponding to the eigenvalue $\frac{1}{\lambda-i \lambda_{0}}$. Hence, the spectrum of the ITE problem is discrete.

Remark 6 As in the proof of Theorem 1, the condition $0 \leq \alpha<2$ in Theorem 2 is necessary to guarantee the compactness of $T_{2}$.

\subsection{On the case $A_{1} \geq A_{2}$ globally in $\Omega$}

The main result of this section is

Proposition 8 Assume that

$$
A_{1}-A_{2} \geq c d_{\Gamma}^{\alpha} I \text { in } \Omega \text { and } \int_{\Omega}\left(\Sigma_{1}-\Sigma_{2}\right) \neq 0,
$$

for some $0 \leq \alpha<2$ and for some $c>0$. The spectrum of (1.3)-(1.4) is discrete.

In Proposition 8 the condition $A_{1}-A_{2} \geq c d_{\Gamma}^{\alpha} I$ is required in the whole domain $\Omega$ not in a neighborhood of $\Gamma$; this is different from the context of Theorem 2. Applying Proposition 8 with $\alpha=0$ and $\left(A_{1}, \Sigma_{1}\right)=(I, 1)$ in $\Omega$, one rediscovers the discreteness result given in [3, Theorem 4.4].

The proof of Proposition 8 is based on the following lemma

Lemma 12 Assume that for some $c>0$ and for some $0 \leq \alpha<2$,

$$
A_{1}-A_{2} \geq c d_{\Gamma}^{\alpha} I \text { in } \Omega \text { and } \int_{\Omega}\left(\Sigma_{1}-\Sigma_{2}\right) \neq 0 .
$$

There exists $\Lambda_{0}>0$ such that if $0<\lambda_{0}<\Lambda_{0}$, and $v=\left(v_{1}, v_{2}\right) \in\left[H_{l o c}^{1}(\Omega)\right]^{2}$ verifies

$$
\left\{\begin{array}{l}
\operatorname{div}\left(A_{1} \nabla v_{1}\right)-i \lambda_{0} \Sigma_{1} v_{1}=g_{1} \text { in } \Omega, \\
\operatorname{div}\left(A_{2} \nabla v_{2}\right)-i \lambda_{0} \Sigma_{2} v_{2}=g_{2} \text { in } \Omega,
\end{array}\right.
$$

and (3.6) and (3.7) for some $g=\left(g_{1}, g_{2}\right) \in\left[L^{2}(\Omega)\right]^{2}$ and $h \in H^{-1 / 2}(\Gamma)$, then

$$
\|v\|_{\mathcal{H}(\Omega)}^{2} \leq C \mathcal{M}(v, g, h)
$$

for some positive constant $C$ independent of $v, g$, and $h$ where $\mathcal{M}(v, g, h)$ is defined in (2.8).

Proof The proof is in the spirit of the one of Lemma 11. From (3.6), we have, for all $0<\gamma<1$

$$
\left.\left.\lambda_{0}\left|\int_{\Omega}\left(\Sigma_{2}-\Sigma_{1}\right)\right| v_{2}\right|^{2}\left|\leq \int_{\Omega} \gamma \lambda_{0}\right| v_{2}\right|^{2}+C \gamma^{-1} \lambda_{0}|w|^{2}+\mathcal{M}(v, g, h) .
$$

Here and in what follows in the proof, $C$ denotes a positive constant depending only on $\Lambda_{1}, \Lambda_{2}, \Omega$; it is independent of $\lambda_{0}$ and $\gamma$. As in (3.23), we deduce from (3.7) that

$$
\int_{\Omega}\left\langle A_{1} \nabla w, \nabla w\right\rangle+\int_{\Omega}\left\langle\left[A_{1}-A_{2}\right] \nabla v_{2}, \nabla v_{2}\right\rangle \leq \int_{\Omega} \gamma \lambda_{0}\left|v_{2}\right|^{2}+C \gamma^{-1} \lambda_{0}|w|^{2}+C \mathcal{M}(v, g, h) \text {. }
$$

Note that $\int_{\Omega}|w|^{2} \leq C \int_{\Omega}|\nabla w|^{2}$ by Poincaré's inequality, and

$$
\int_{\Omega}\left|v_{2}\right|^{2} \leq\left.\left. C\left|\int_{\Omega}\left(\Sigma_{2}-\Sigma_{1}\right)\right| v_{2}\right|^{2}\left|+C \int_{\Omega} d_{\Gamma}^{\alpha}\right| \nabla v_{2}\right|^{2}
$$


by Lemma 4. Fixing $\gamma$ small enough in (3.45) and (3.46), adding (3.45) and (3.46), and using (3.47), we derive that if $\lambda_{0}$ is small enough,

$$
\int_{\Omega}\left\langle A_{1} \nabla w, \nabla w\right\rangle+\int_{\Omega}\left\langle\left[A_{1}-A_{2}\right] \nabla v_{2}, \nabla v_{2}\right\rangle+\lambda_{0} \int_{\Omega}\left|v_{2}\right|^{2} \leq C \mathcal{M}(v, g, h) .
$$

The conclusion now follows from (3.48) by noting that $w=v_{1}-v_{2}$.

We are ready to present

Proof of Proposition 8 The proof follows as in the one of Theorem 2 by considering the transformation

$$
\begin{aligned}
T_{2}:\left[L^{2}(\Omega)\right]^{2} & \rightarrow\left[L^{2}(\Omega)\right]^{2} \\
\left(f_{1}, f_{2}\right) & \mapsto\left(u_{1}, u_{2}\right),
\end{aligned}
$$

where $\left(u_{1}, u_{2}\right) \in \mathcal{H}(\Omega)$ is the unique viscosity solution of (3.1). The details are left to the reader.

\section{On the case $A_{1}=A_{2}$ in a neighborhood of $\Gamma$ : Proof of Theorem 3}

The condition $A_{1}-A_{2} \geq c d_{\Gamma}^{\alpha} I$ in a neighborhood of $\Gamma$ with $0 \leq \alpha<2$ plays a crucial role in establishing the compactness of $T$, more precisely $T_{1}$ and $T_{2}$ (see Remarks 4 and 6). To be able to deal with case $A_{1}=A_{2}$ in a neighborhood of $\Gamma$, we make some modifications on $T$. The idea is to take into account the fact that $u_{1}-u_{2} \in H_{0}^{1}(\Omega)$ which is more regular $u_{1}$ and $u_{2}$ which are in general not in $H^{1}(\Omega)$. A modification on $T$ was also used in the work of Sylvester [29]. Nevertheless, the modification in [29] requires the condition $A_{1}=A_{2}$ globally in $\Omega$ and does not fit in our situation.

The motivation for reformulating the problem is as follows. Let $\lambda \in \mathbb{C}$ be an eigenvalue of the ITE problem and let $\left(u_{1}, u_{2}\right) \in\left[H^{1}(\Omega)\right]^{2}$ be a corresponding pair of eigenfunctions. Then

$$
\begin{cases}\operatorname{div}\left(A_{1} \nabla u_{1}\right)-\lambda \Sigma_{1} u_{1}=0 & \text { in } \Omega, \\ \operatorname{div}\left(A_{2} \nabla u_{2}\right)-\lambda \Sigma_{2} u_{2}=0 & \text { in } \Omega, \\ u_{1}=u_{2}, \quad A_{1} \nabla u_{1} \cdot v=A_{2} \nabla u_{2} \cdot v & \text { on } \Gamma .\end{cases}
$$

Fix $\lambda_{0} \neq 0$ and set $w=u_{1}-u_{2}$ in $\Omega$. From (4.1), we have

$$
\begin{aligned}
\operatorname{div}\left(A_{1} \nabla w\right)-\lambda_{0} \Sigma_{1} w= & \left(\lambda-\lambda_{0}\right) \Sigma_{1} u_{1}-\operatorname{div}\left(A_{1} \nabla u_{2}\right)+\lambda_{0} \Sigma_{1} u_{2} \\
= & \left(\lambda-\lambda_{0}\right) \Sigma_{1}\left(u_{1}-u_{2}\right)-\lambda \Sigma_{2} u_{2} \\
& -\operatorname{div}\left(\left[A_{1}-A_{2}\right] \nabla u_{2}\right)+\lambda \Sigma_{1} u_{2} \quad \text { in } \Omega .
\end{aligned}
$$

Define $^{4}$

$$
\hat{w}=w+\lambda u_{2} / \lambda_{0} \quad \text { in } \Omega .
$$

Then

$$
\begin{aligned}
\operatorname{div}\left(A_{1} \nabla \hat{w}\right)-\lambda_{0} \Sigma_{1} \hat{w}= & \left(\lambda-\lambda_{0}\right) \Sigma_{1}\left(u_{1}-u_{2}\right)+\frac{\lambda}{\lambda_{0}}\left(\lambda-\lambda_{0}\right) \Sigma_{2} u_{2} \\
& +\frac{\lambda-\lambda_{0}}{\lambda_{0}} \operatorname{div}\left(\left[A_{1}-A_{2}\right] \nabla u_{2}\right) \text { in } \Omega .
\end{aligned}
$$

4 The goal is to eliminate $\Sigma_{1} u_{2}$ from the equation of $\hat{w}$. 
It is clear that

$$
\operatorname{div}\left(A_{2} \nabla u_{2}\right)-\lambda_{0} \Sigma_{2} u_{2}=\left(\lambda-\lambda_{0}\right) \Sigma_{2} u_{2} \quad \text { in } \Omega .
$$

Set

$$
U_{1}=\hat{w}=\left(u_{1}-u_{2}\right)+\lambda u_{2} / \lambda_{0} \quad \text { and } \quad U_{2}=\lambda u_{2} / \lambda_{0} \quad \text { in } \Omega .
$$

It follows from (4.2) and (4.3) that, in $\Omega$,

$$
\begin{aligned}
& \operatorname{div}\left(A_{1} \nabla U_{1}\right)-\lambda_{0} \Sigma_{1} U_{1} \\
& \quad=\left(\lambda-\lambda_{0}\right) \Sigma_{1}\left(U_{1}-U_{2}\right)+\left(\lambda-\lambda_{0}\right) \Sigma_{2} U_{2}+\frac{\lambda-\lambda_{0}}{\lambda} \operatorname{div}\left(\left[A_{1}-A_{2}\right] \nabla U_{2}\right)
\end{aligned}
$$

and

$$
\operatorname{div}\left(A_{2} \nabla U_{2}\right)-\lambda_{0} \Sigma_{2} U_{2}=\left(\lambda-\lambda_{0}\right) \Sigma_{2} U_{2} .
$$

Since $A_{1}=A_{2}$ near $\Gamma$, we also have

$$
U_{1}=U_{2}, \quad A_{1} \nabla U_{1} \cdot v=A_{2} \nabla U_{2} \cdot v \text { on } \Gamma .
$$

One can rewrite (4.4), (4.5), and (4.6) under the form

$$
T_{3, \lambda}\left(U_{1}, U_{2}\right)=\frac{1}{\lambda-\lambda_{0}}\left(U_{1}, U_{2}\right) .
$$

Here

$$
T_{3, \lambda}\left(f_{1}, f_{2}\right):=\left(v_{1}, v_{2}\right)
$$

where $\left(v_{1}, v_{2}\right)$ satisfies the system

$$
\begin{cases}\operatorname{div}\left(A_{1} \nabla v_{1}\right)-\lambda_{0} \Sigma_{1} v_{1}=\Sigma_{1}\left(f_{1}-f_{2}\right)+\Sigma_{2} f_{2}+\lambda^{-1} \operatorname{div}\left(\left[A_{1}-A_{2}\right] \nabla f_{2}\right) & \text { in } \Omega, \\ \operatorname{div}\left(A_{2} \nabla v_{2}\right)-\lambda_{0} \Sigma_{2} v_{2}=\Sigma_{2} f_{2} & \text { in } \Omega, \\ v_{1}=v_{2}, \quad A_{1} \nabla v_{1} \cdot v=A_{2} \nabla v_{2} \cdot v & \text { on } \Gamma .\end{cases}
$$

The discreteness of $T$ can now be derived from the discreteness of $T_{3, \lambda}$. To this end, we first establish the well-posedness of the system

$$
\begin{cases}\operatorname{div}\left(A_{1} \nabla v_{1}\right)-\lambda_{0} \Sigma_{1} v_{1}=g_{1}+\operatorname{div}\left(G_{1}\right) & \text { in } \Omega, \\ \operatorname{div}\left(A_{2} \nabla v_{2}\right)-\lambda_{0} \Sigma_{2} v_{2}=g_{2} & \text { in } \Omega, \\ v_{1}=v_{2}, \quad A_{1} \nabla v_{1} \cdot v=A_{2} \nabla v_{2} \cdot v & \text { on } \Gamma,\end{cases}
$$

for appropriate functions $g_{1}, g_{2}$ and vector field $G_{1}$. We follows the strategy used in the proof of Theorem 1. We first introduce some notations. Given $s \in \mathbb{R}$, we denote

$$
L^{2}\left(\Omega, d_{\Gamma}^{s}\right):=\left\{\psi \in L_{l o c}^{2}(\Omega) ;\|\psi\|_{L^{2}\left(\Omega, d_{\Gamma}^{s}\right)}<+\infty\right\},
$$

where

$$
\|\psi\|_{L^{2}\left(\Omega, d_{\Gamma}^{s}\right)}^{2}:=\int_{\Omega} d_{\Gamma}^{s}|\psi|^{2} .
$$

We also define, for a given $0 \leq \beta_{1}<2$,

$$
\mathcal{H}_{0}(\Omega)=\left\{\left(\psi_{1}, \psi_{2}\right) \in L_{l o c}^{2}(\Omega) ; \psi_{1}, \psi_{2} \in L^{2}\left(\Omega, d_{\Gamma}^{\beta_{1}}\right) \text { and } \psi_{1}-\psi_{2} \in L^{2}\left(\Omega, d_{\Gamma}^{-\beta_{1}}\right)\right\}
$$

and

$$
\left\|\left(\psi_{1}, \psi_{2}\right)\right\|_{\mathcal{H}_{0}(\Omega)}:=\left\|\left(\psi_{1}, \psi_{2}\right)\right\|_{L^{2}(\Omega)}+\left\|\psi_{1}-\psi_{2}\right\|_{L^{2}\left(\Omega, d_{\Gamma}^{-\beta_{1}}\right)}
$$

Here is a variant of Lemma 1. 
Lemma 13 Let $0 \leq \beta_{1}<2, \lambda_{0}>0, g=\left(g_{1}, g_{2}\right) \in \mathcal{H}_{0}(\Omega)$, and $G_{1} \in\left[L^{2}(\Omega)\right]^{d}$ with $\operatorname{supp} G_{1} \subset \subset \Omega$. Assume that $v=\left(v_{1}, v_{2}\right) \in\left[H^{1}(\Omega)\right]^{2}$ satisfy the system

$$
\begin{cases}\operatorname{div}\left(A_{1} \nabla v_{1}\right)-\lambda_{0} \Sigma_{1} v_{1}=g_{1}+\operatorname{div}\left(G_{1}\right) & \text { in } \Omega, \\ \operatorname{div}\left(A_{2} \nabla v_{2}\right)-\lambda_{0} \Sigma_{2} v_{2}=g_{2} & \text { in } \Omega, \\ v_{1}=v_{2}, \quad A_{1} \nabla v_{1} \cdot v=A_{2} \nabla v_{2} \cdot v & \text { on } \Gamma .\end{cases}
$$

We have, with $w=v_{1}-v_{2}$,

$$
\begin{aligned}
& \int_{\Omega}\left\langle A_{1} \nabla w, \nabla w\right\rangle+\lambda_{0} \Sigma_{1}|w|^{2} \\
& =-\int_{\Omega}\left(g_{1}-g_{2}\right) \bar{w}+\int_{\Omega}\left\langle G_{1}, \nabla \bar{w}\right\rangle+\int_{\Omega} \lambda_{0}\left(\Sigma_{2}-\Sigma_{1}\right) v_{2} \bar{w} \\
& \quad+\int_{\Omega}\left\langle\left[A_{2}-A_{1}\right] \nabla v_{2}, \nabla w\right\rangle
\end{aligned}
$$

and

$$
\begin{gathered}
\int_{\Omega}\left\langle\left[A_{1}-A_{2}\right] \nabla v_{2}, \nabla w\right\rangle+\left\langle\left[A_{1}-A_{2}\right] \nabla v_{2}, \nabla v_{2}\right\rangle+\lambda_{0}\left(\Sigma_{1}-\Sigma_{2}\right)\left|v_{2}\right|^{2} \\
=\int_{\Omega} g_{2} \bar{w}-\left(\bar{g}_{1}-\bar{g}_{2}\right) v_{2}+\lambda_{0}\left(\Sigma_{2}-\Sigma_{1}\right) v_{2} \bar{w}+\int_{\Omega}\left\langle\nabla v_{2}, G_{1}\right\rangle .
\end{gathered}
$$

As a consequence of (4.10) and (4.11), we obtain

$$
\begin{aligned}
\int_{\Omega}\left\langle A_{1} \nabla w, \nabla w\right\rangle+\lambda_{0} \Sigma_{1}|w|^{2} \leq & 4 \mathcal{N}\left(v, g, G_{1}\right)+\int_{\Omega}\left|\left\langle\left[A_{2}-A_{1}\right] \nabla v_{2}, \nabla w\right\rangle\right| \\
& +\lambda_{0}\left|\left(\Sigma_{2}-\Sigma_{1}\right) v_{2} \bar{w}\right|
\end{aligned}
$$

and

$$
\begin{aligned}
& \int_{\Omega}\left\langle\left[A_{1}-A_{2}\right] \nabla v_{2}, \nabla v_{2}\right\rangle+\lambda_{0}\left(\Sigma_{1}-\Sigma_{2}\right)\left|v_{2}\right|^{2} \\
& \quad \leq 4 \mathcal{N}\left(v, g, G_{1}\right)+\int_{\Omega}\left|\left\langle\left[A_{2}-A_{1}\right] \nabla v_{2}, \nabla w\right\rangle\right|+\lambda_{0}\left|\left(\Sigma_{2}-\Sigma_{1}\right) v_{2} \bar{w}\right|,
\end{aligned}
$$

where

$$
\mathcal{N}\left(v, g, G_{1}\right)=\int_{\Omega}|g||w|+\left|g_{1}-g_{2}\right||v|+\left|G_{1}\right|\left|\nabla v_{2}\right| .
$$

Proof The proof of Lemma 13 follows closely to the one of Lemma 1 by noting that one still has (2.4) and (2.5) in which $g_{1}$ is replaced by $g_{1}+\operatorname{div}\left(G_{1}\right)$ and $h=0$, i.e, (4.10) and (4.11) hold. The details are left to the reader.

We next establish a variant of Lemma 5.

Lemma 14 Let $0 \leq \beta<\beta_{1}<2$ and assume that, for some $c, \tau>0$,

$$
A_{1} \geq A_{2} \quad \text { and } \quad \Sigma_{1}-\Sigma_{2} \geq c d_{\Gamma}^{\beta} \text { in } \Omega_{\tau} .
$$

There exists $\Lambda_{0}>1$ such that if $\lambda_{0} \geq \Lambda_{0}$, and $v=\left(v_{1}, v_{2}\right) \in\left[H_{l o c}^{1}(\Omega)\right]^{2}$ satisfies the system

$$
\begin{cases}\operatorname{div}\left(A_{1} \nabla v_{1}\right)-\lambda_{0} \Sigma_{1} v_{1}=g_{1}+\operatorname{div}\left(G_{1}\right) & \text { in } \Omega, \\ \operatorname{div}\left(A_{2} \nabla v_{2}\right)-\lambda_{0} \Sigma_{2} v_{2}=g_{2} & \text { in } \Omega,\end{cases}
$$


and (4.12) and (4.13) for some $g=\left(g_{1}, g_{2}\right) \in \mathcal{H}_{0}(\Omega)$, and $G_{1} \in\left[L^{2}(\Omega)\right]^{d}$ with supp $G_{1} \subset \subset$ $\Omega$, then, for $w=v_{1}-v_{2}$ in $\Omega$,

$$
\begin{aligned}
& \int_{\Omega_{\tau}}\left\langle\left[A_{1}-A_{2}\right] \nabla v_{2}, \nabla v_{2}\right\rangle+\int_{\Omega_{\tau}}\left(\Sigma_{1}-\Sigma_{2}\right)\left|v_{2}\right|^{2}+\int_{\Omega \backslash \Omega_{\tau}}\left(\left|\nabla v_{2}\right|^{2}+\left|v_{2}\right|^{2}\right)+\int_{\Omega}|\nabla w|^{2} \\
& \quad \leq C\left(\mathcal{N}\left(v, g, G_{1}\right)+\left\|g_{2}\right\|_{L^{2}\left(\Omega \backslash \Omega_{\tau / 2}\right)}^{2}\right),
\end{aligned}
$$

for some positive constant $C$ independent of $v, g$, and $G_{1}$ where $\mathcal{N}\left(v, g, G_{1}\right)$ is defined by (4.14). In particular, if $v \in\left[H^{1}(\Omega)\right]^{2}$ is a solution of (4.9) then (4.17) holds.

Proof The proof is in the spirit of the one of Lemma 5 and even simpler. Adding (4.12) and (4.13), using the fact, for $\gamma>0$,

$\gamma \int_{\Omega_{\tau}}\left\langle\left[A_{1}-A_{2}\right] \nabla w, \nabla w\right\rangle+\frac{1}{\gamma} \int_{\Omega_{\tau}}\left\langle\left[A_{1}-A_{2}\right] \nabla v_{2}, \nabla v_{2}\right\rangle \geq 2 \int_{\Omega_{\tau}}\left|\left\langle\left[A_{1}-A_{2}\right] \nabla w, \nabla v_{2}\right\rangle\right|$ and

$$
\gamma \int_{\Omega_{\tau}}\left(\Sigma_{1}-\Sigma_{2}\right)|w|^{2}+\frac{1}{\gamma} \int_{\Omega_{\tau}}\left(\Sigma_{1}-\Sigma_{2}\right)\left|v_{2}\right|^{2} \geq 2 \int_{\Omega_{\tau}}\left(\Sigma_{1}-\Sigma_{2}\right)\left|w v_{2}\right|,
$$

and taking $\gamma>1$ and close to 1 in the previous two inequalities, we obtain

$$
\begin{aligned}
& \int_{\Omega}|\nabla w|^{2}+\lambda_{0}|w|^{2}+\lambda_{0}\left(\Sigma_{1}-\Sigma_{2}\right)\left|v_{2}\right|^{2}+\int_{\Omega_{\tau}}\left\langle\left[A_{1}-A_{2}\right] \nabla v_{2}, \nabla v_{2}\right\rangle \\
& \quad \leq C \mathcal{N}\left(v, g, G_{1}\right)+C \int_{\Omega \backslash \Omega_{\tau}}\left|\nabla v_{2}\right|^{2}+\lambda_{0}\left|v_{2} w\right|,
\end{aligned}
$$

for some positive constant $C$ independent of $v, g, G_{1}$, and $\lambda_{0}$. Since, for $\gamma>0$,

$$
2\left|v_{2} w\right| \leq \gamma\left|v_{2}\right|^{2}+\gamma^{-1}|w|^{2}
$$

by applying Lemma 2 to $v_{2}$ in $\Omega \backslash \Omega_{\tau / 2}$ after fixing $\gamma>1$ large enough in (4.19), we have, for large $\lambda_{0}$,

$$
\begin{aligned}
& \int_{\Omega_{\tau}}\left\langle\left[A_{1}-A_{2}\right] \nabla v_{2}, \nabla v_{2}\right\rangle+\int_{\Omega_{\tau}}\left(\Sigma_{1}-\Sigma_{2}\right)\left|v_{2}\right|^{2}+\int_{\Omega \backslash \Omega_{\tau}}\left(\left|\nabla v_{2}\right|^{2}+\left|v_{2}\right|^{2}\right)+\int_{\Omega}|\nabla w|^{2} \\
& \quad \leq C\left(\mathcal{N}\left(v, g, G_{1}\right)+\left\|g_{2}\right\|_{L^{2}\left(\Omega \backslash \Omega_{\tau / 2}\right)}^{2}\right),
\end{aligned}
$$

for some positive constant $C$ independent of $v, g$, and $G_{1}$; which is the conclusion.

We now introduce functional spaces. In what follows in this section, we assume that (1.12) holds in $\Omega_{\tau}$ for some $\tau>0$ and set $\beta_{1}=(2+\beta) / 2$. Define

$$
\hat{\mathcal{H}}_{1}(\Omega):=\left\{\left(v_{1}, v_{2}\right) \in\left[H_{l o c}^{1}(\Omega)\right]^{2} ; v_{1}-v_{2} \in H_{0}^{1}(\Omega),\left\|\left(v_{1}, v_{2}\right)\right\|_{\hat{\mathcal{H}}_{1}(\Omega)}<+\infty\right\},
$$

where the norm is generated from the following scalar product, with $u=\left(u_{1}, u_{2}\right)$ and $v=\left(v_{1}, v_{2}\right)$,

$$
\langle u, v\rangle_{\hat{\mathcal{H}}_{1}(\Omega)}=\int_{\Omega} \nabla\left(u_{1}-u_{2}\right) \nabla\left(\bar{v}_{1}-\bar{v}_{2}\right)+\int_{\Omega} d_{\Gamma}^{\beta_{1}+2} \nabla u_{2} \nabla \bar{v}_{2}+\int_{\Omega \backslash \Omega_{\tau}} u \bar{v}+\int_{\Omega_{\tau}}\left(\Sigma_{1}-\Sigma_{2}\right) u \bar{v}
$$

One can verify that $\hat{\mathcal{H}}_{1}(\Omega)$ is a Hilbert space.

We are ready to give the 
Definition 5 Let $\left(g_{1}, g_{2}\right) \in \mathcal{H}_{0}(\Omega)$ and $G_{1} \in\left[L^{2}(\Omega)\right]^{d}$ such that supp $G_{1} \subset \Omega \backslash \Omega_{\tau}$. A pair of functions $\left(v_{1}, v_{2}\right) \in \hat{\mathcal{H}}_{1}(\Omega)$ is called a weak solution of (4.8) if

$$
\begin{cases}\operatorname{div}\left(A_{1} \nabla v_{1}\right)-\lambda_{0} \Sigma_{1} v_{1}=g_{1}+\operatorname{div}\left(G_{1}\right) & \text { in } \Omega, \\ \operatorname{div}\left(A_{2} \nabla v_{2}\right)-\lambda_{0} \Sigma_{2} v_{2}=g_{2} & \text { in } \Omega, \\ \left(A_{1} \nabla v_{1}-A_{2} \nabla v_{2}\right) \cdot v=0 & \text { on } \Gamma .\end{cases}
$$

Remark 7 Since $\operatorname{div}\left(A_{1} \nabla v_{1}-A_{2} \nabla v_{2}\right) \in L^{2}\left(\Omega \backslash \Omega_{\tau}\right)$ and $A_{1} \nabla v_{1}-A_{2} \nabla v_{2}=A_{1} \nabla\left(v_{1}-\right.$ $\left.v_{2}\right)+\left(A_{1}-A_{2}\right) \nabla v_{2} \in L^{2}(\Omega)$ by (4.21). The last identity on $\Gamma$ in (4.22) makes sense.

Applying Lemma 14, we can prove

Lemma 15 Let $g=\left(g_{1}, g_{2}\right) \in \mathcal{H}_{0}(\Omega)$ and $G_{1} \in L^{2}(\Omega)$ with supp $G_{1} \subset \Omega \backslash \Omega_{\tau}$. There exists $\Lambda_{0}>1$ such that if $\lambda_{0} \geq \Lambda_{0}$, then for all $0<\delta<1$, there exists a unique solution $v_{\delta}=\left(v_{1, \delta}, v_{2, \delta}\right) \in\left[H^{1}(\Omega)\right]^{2}$ satisfy the system

$$
\begin{cases}\operatorname{div}\left((1+\delta) A_{1} \nabla v_{1, \delta}\right)-\lambda_{0}(1+\delta) \Sigma_{1} v_{1, \delta}=g_{1}+\operatorname{div}\left(G_{1}\right) & \text { in } \Omega \\ \operatorname{div}\left(A_{2} \nabla v_{2, \delta}\right)-\lambda_{0} \Sigma_{2} v_{2, \delta}=g_{2} & \text { in } \Omega \\ v_{1, \delta}=v_{2, \delta}, \quad(1+\delta) A_{1} \nabla v_{1, \delta} \cdot v=A_{2} \nabla v_{2, \delta} \cdot v & \text { on } \Gamma\end{cases}
$$

Moreover,

$$
\int_{\Omega} \delta\left(\left|\nabla v_{2, \delta}\right|^{2}+\left|v_{2, \delta}\right|^{2}\right)+\left\|v_{\delta}\right\|_{\hat{\mathcal{H}}_{1}(\Omega)}^{2} \leq C\left(\|g\|_{\mathcal{H}_{0}(\Omega)}^{2}+\left\|G_{1}\right\|_{L^{2}(\Omega)}^{2}\right),
$$

for some positive constant $C$ independent of $\delta, g$, and $G_{1}$. As a consequence, there exists a weak solution $v=\left(v_{1}, v_{2}\right) \in \hat{\mathcal{H}}_{1}(\Omega)$ of $(4.8)$ such that

$$
\|v\|_{\hat{\mathcal{H}}_{1}(\Omega)} \leq C\left(\|g\|_{\mathcal{H}_{0}(\Omega)}+\left\|G_{1}\right\|_{L^{2}(\Omega)}^{2}\right) .
$$

Proof Assume that $v_{\delta}=\left(v_{1, \delta}, v_{2, \delta}\right) \in\left[H^{1}(\Omega)\right]^{2}$ is a solution of (4.23). Set $w_{\delta}=v_{1, \delta}-v_{2, \delta}$. Applying Lemma 14 to $\left(A_{1}, A_{2}\right)=\left((1+\delta) A_{1}, A_{2}\right)$ and $\left(\Sigma_{1}, \Sigma_{2}\right)=\left((1+\delta) \Sigma_{1}, \Sigma_{2}\right)$, one obtains, for large $\lambda_{0}$,

$$
\begin{aligned}
& \int_{\Omega_{\tau}}\left\langle\left[(1+\delta) A_{1}-A_{2}\right] \nabla v_{2, \delta}, \nabla v_{2, \delta}\right\rangle+\int_{\Omega_{\tau}}\left((1+\delta) \Sigma_{1}-\Sigma_{2}\right)\left|v_{2, \delta}\right|^{2} \\
& \quad+\int_{\Omega \backslash \Omega_{\tau}}\left(\left|\nabla v_{2, \delta}\right|^{2}+\left|v_{2, \delta}\right|^{2}\right)+\int_{\Omega}\left|\nabla w_{\delta}\right|^{2} \\
& \leq C\left(\mathcal{N}\left(v_{\delta}, g, G_{1}\right)+\left\|g_{2}\right\|_{L^{2}\left(\Omega \backslash \Omega_{\tau / 2}\right)}^{2}\right) .
\end{aligned}
$$

We have

$$
\begin{aligned}
\int_{\Omega}|g|\left|w_{\delta}\right| & \leq\left(\int_{\Omega} d_{\Gamma}^{\beta_{1}}|g|^{2}\right)^{1 / 2}\left(\int_{\Omega} d_{\Gamma}^{-\beta_{1}}\left|w_{\delta}\right|^{2}\right)^{1 / 2}, \\
\int_{\Omega}\left|g_{1}-g_{2}\right|\left|v_{\delta}\right| & \leq\left(\int_{\Omega} d_{\Gamma}^{-\beta_{1}}\left|g_{1}-g_{2}\right|^{2}\right)^{1 / 2}\left(\int_{\Omega} d_{\Gamma}^{\beta_{1}}\left|v_{\delta}\right|^{2}\right)^{1 / 2}, \\
\int_{\Omega}\left|G_{1}\right|\left|\nabla v_{2, \delta}\right| & \leq\left(\int_{\Omega \backslash \Omega_{\tau}}\left|G_{1}\right|^{2}\right)^{1 / 2}\left(\int_{\Omega \backslash \Omega_{\tau}}\left|\nabla v_{2, \delta}\right|^{2}\right)^{1 / 2} .
\end{aligned}
$$

Noting that $w_{\delta} \in H_{0}^{1}(\Omega)$ and hence, by the Hardy inequality, we obtain

$$
\int_{\Omega} d_{\Gamma}^{-2}\left|w_{\delta}\right|^{2} \leq C_{\Omega} \int_{\Omega}\left|\nabla w_{\delta}\right|^{2}
$$


Since $0 \leq \beta<\beta_{1}<2$, it follows from the definition of $\mathcal{N}$ that

$$
\mathcal{N}\left(v_{\delta}, g, G_{1}\right) \leq C\left(\|g\|_{\mathcal{H}_{0}(\Omega)}+\left\|G_{1}\right\|_{L^{2}\left(\Omega \backslash \Omega_{\tau}\right)}\right)\left\|v_{\delta}\right\|_{\hat{\mathcal{H}}_{1}(\Omega)} .
$$

By Lemma 6, we have

$$
\int_{\Omega} d_{\Gamma}^{\beta+2}\left|\nabla v_{2}\right|^{2} \leq C \int_{\Omega} d_{\Gamma}^{\beta}\left|v_{2}\right|^{2}+d_{\Gamma}^{\beta}\left|g_{2}\right|^{2} .
$$

Since $\beta_{1}>\beta$, we derive from (4.28) that

$$
\begin{aligned}
& \int_{\Omega_{\tau}}\left\langle\left[(1+\delta) A_{1}-A_{2}\right] \nabla v_{2, \delta}, \nabla v_{2, \delta}\right\rangle+\int_{\Omega_{\tau}}\left((1+\delta) \Sigma_{1}-\Sigma_{2}\right)\left|v_{2, \delta}\right|^{2} \\
& \quad+\int_{\Omega \backslash \Omega_{\tau}}\left(\left|\nabla v_{2, \delta}\right|^{2}+\left|v_{2, \delta}\right|^{2}\right)+\int_{\Omega}|\nabla w|^{2}+\|g\|_{\mathcal{H}_{0}(\Omega)}^{2} \\
& \quad \geq C\left(\left\|v_{\delta}\right\|_{\hat{\mathcal{H}}_{1}(\Omega)}^{2}+\int_{\Omega} \delta\left(\left|\nabla v_{2, \delta}\right|^{2}+\left|v_{2, \delta}\right|^{2}\right)\right)
\end{aligned}
$$

Combining (4.26), (4.27), and (4.29) yields (4.24). This in turn implies the uniqueness of $v_{\delta}$. The existence of $v_{\delta}$ can be established via an approximation argument by first assuming that $g \in\left[L^{2}(\Omega)\right]^{2}$ and $G_{1} \in\left[L^{2}(\Omega)\right]^{d}$ and then considering the general case; the existence in first case follows from Proposition $4^{5}$. The details of this fact are left to the reader. The existence and estimate of a weak solution $v \in \hat{\mathcal{H}}_{1}(\Omega)$ of (4.8) follows directly from the existence and the estimate of $v_{\delta}$. The details are omitted.

As in the proof of Theorem 1 in Sect. 2, we introduce the concept of viscosity solutions.

Definition 6 Let $\left(g_{1}, g_{2}\right) \in \mathcal{H}_{0}(\Omega)$ and $G_{1} \in L^{2}(\Omega)$ with supp $G_{1} \subset \Omega \backslash \Omega_{\tau}$. A weak solution $\left(v_{1}, v_{2}\right) \in \hat{\mathcal{H}}_{1}(\Omega)$ of $(4.8)$ is called a viscosity solution if for any $\left(\hat{v}_{1}, \hat{v}_{2}\right) \in$ $\hat{\mathcal{H}}_{1}(\Omega),\left(\hat{g}_{1}, \hat{g}_{2}\right) \in \mathcal{H}_{0}(\Omega)$, and $\hat{G}_{1} \in\left[L^{2}(\Omega)\right]^{d}$ with supp $\hat{G}_{1} \subset \Omega \backslash \Omega_{\tau}$ such that

$$
\begin{cases}\operatorname{div}\left(A_{1} \nabla \hat{v}_{1}\right)-\lambda_{0} \Sigma_{1} \hat{v}_{1}=\hat{g}_{1}+\operatorname{div}\left(\hat{G}_{1}\right) & \text { in } \Omega, \\ \operatorname{div}\left(A_{2} \nabla \hat{v}_{2}\right)-\lambda_{0} \Sigma_{2} \hat{v}_{2}=\hat{g}_{2} & \text { in } \Omega, \\ A_{1} \nabla \hat{v}_{1} \cdot v-A_{2} \nabla \hat{v}_{2} \cdot v=0 & \text { on } \Gamma .\end{cases}
$$

we have, with $w=v_{1}-v_{2}$ and $\hat{w}=\hat{v}_{1}-\hat{v}_{2}$,

$$
\begin{aligned}
& \int_{\Omega}\left\langle A_{1} \nabla w, \nabla \hat{w}\right\rangle+\lambda_{0} \Sigma_{1} w \overline{\hat{w}}=-\int_{\Omega}\left(g_{1}-g_{2}\right) \overline{\hat{w}}+\int_{\Omega} \lambda_{0}\left(\Sigma_{2}-\Sigma_{1}\right) v_{2} \overline{\hat{w}} \\
& \quad+\int_{\Omega}\left\langle\left[A_{2}-A_{1}\right] \nabla v_{2}, \nabla \hat{w}\right\rangle+\int_{\Omega} G_{1} \nabla \overline{\hat{w}}
\end{aligned}
$$

and

$$
\begin{aligned}
& \int_{\Omega}\left\langle\left[A_{1}-A_{2}\right] \nabla v_{2}, \nabla \hat{w}\right\rangle+\left\langle\left[A_{1}-A_{2}\right] \nabla v_{2}, \nabla \hat{v}_{2}\right\rangle+\lambda_{0}\left(\Sigma_{1}-\Sigma_{2}\right) v_{2} \overline{\hat{v}}_{2} \\
& \quad=\int_{\Omega} g_{2} \overline{\hat{w}}-\left(\overline{\hat{g}}_{1}-\overline{\hat{g}}_{2}\right) v_{2}+\overline{\hat{G}}_{1} \nabla v_{2}+\lambda_{0}\left(\Sigma_{2}-\Sigma_{1}\right) v_{2} \overline{\hat{w}} .
\end{aligned}
$$

We are now in a position to state and prove the key result of this section.

5 In Proposition 4, $G_{1}=0$; nevertheless, the same proof gives the same result in the case $G_{1} \in L^{2}(\Omega)$ with $\operatorname{supp} G_{1} \subset \subset \Omega$. 
Proposition 9 There exists $\Lambda_{0}>1$ such that for $\lambda_{0}>\Lambda_{0}$ and for $g=\left(g_{1}, g_{2}\right) \in \mathcal{H}_{0}(\Omega)$ and $G_{1} \in\left[L^{2}(\Omega)\right]^{d}$ with supp $G_{1} \subset \Omega \backslash \Omega_{\tau}$, there exists a unique viscosity solution $v=$ $\left(v_{1}, v_{2}\right) \in \hat{\mathcal{H}}_{1}(\Omega)$ of $(4.8)$. Moreover,

$$
\|v\|_{\hat{\mathcal{H}}_{1}(\Omega)} \leq C\left(\|g\|_{\mathcal{H}_{0}(\Omega)}+\left\|G_{1}\right\|_{L^{2}(\Omega)}\right),
$$

for some positive constant $C$ independent of $g$ and $G_{1}$.

Proof We first prove that viscosity solutions exist. By Lemma 15, there exists a unique solution $v_{\delta}=\left(v_{1, \delta}, v_{2, \delta}\right) \in\left[H^{1}(\Omega)\right]^{2}(0<\delta<1)$ of the system

$$
\begin{cases}\operatorname{div}\left((1+\delta) A_{1} \nabla v_{1, \delta}\right)-\lambda_{0}(1+\delta) \Sigma_{1} v_{1, \delta}=g_{1}+\operatorname{div}\left(G_{1}\right) & \text { in } \Omega \\ \operatorname{div}\left(A_{2} \nabla v_{2, \delta}\right)-\lambda_{0} \Sigma_{2} v_{2, \delta}=g_{2} & \text { in } \Omega \\ v_{1, \delta}=v_{2, \delta}, \quad(1+\delta) A_{1} \nabla v_{1, \delta} \cdot v=A_{2} \nabla v_{2, \delta} \cdot v & \text { on } \Gamma\end{cases}
$$

moreover,

$$
\int_{\Omega} \delta\left(\left|\nabla v_{2, \delta}\right|^{2}+\left|v_{2, \delta}\right|^{2}\right)+\left\|v_{\delta}\right\|_{\hat{\mathcal{H}}_{1}(\Omega)}^{2} \leq C\left(\|g\|_{\mathcal{H}_{0}(\Omega)}^{2}+\left\|G_{1}\right\|_{L^{2}(\Omega)}^{2}\right)
$$

for some positive constant $C$ independent of $\delta, g$, and $G_{1}$.

Set $w_{\delta}=v_{1, \delta}-v_{2, \delta}$. Let $v$ be the weak limit of $\left(v_{\delta_{n}}\right)$ in $\hat{\mathcal{H}}_{1}(\Omega)$ for some sequence $\left(\delta_{n}\right) \rightarrow 0$ such that $w_{\delta_{n}} \rightarrow w:=v_{1}-v_{2}$ weakly in $H_{0}^{1}(\Omega)$. Then $v$ is a weak solution of (4.8). We prove that $v$ is a viscosity solution. Multiplying the equation of $w_{\delta}$,

$$
\begin{aligned}
& \operatorname{div}\left((1+\delta) A_{1} \nabla w_{\delta}\right)-\lambda_{0}(1+\delta) \Sigma_{1} w_{\delta} \\
& \quad=g_{1}+\operatorname{div}\left(G_{1}\right)-g_{2}-\lambda_{0}\left(\Sigma_{2}-(1+\delta) \Sigma_{1}\right) v_{2, \delta}+\operatorname{div}\left(\left[A_{2}-(1+\delta) A_{1}\right] \nabla v_{2, \delta}\right) \text { in } \Omega,
\end{aligned}
$$

by $\overline{\hat{w}}$ and integrating in $\Omega$, we obtain

$$
\begin{aligned}
& \int_{\Omega}\left\langle(1+\delta) A_{1} \nabla w_{\delta}, \nabla \hat{w}\right\rangle+\lambda_{0}(1+\delta) \Sigma_{1} w_{\delta} \overline{\hat{w}}=-\int_{\Omega}\left(g_{1}-g_{2}\right) \overline{\hat{w}} \\
& \quad+\int_{\Omega} \lambda_{0}\left(\Sigma_{2}-(1+\delta) \Sigma_{1}\right) v_{2, \delta} \overline{\hat{w}}+\int_{\Omega}\left\langle\left[A_{2}-(1+\delta) A_{1}\right] \nabla v_{2, \delta}, \nabla \hat{w}\right\rangle+\int_{\Omega} G_{1} \nabla \overline{\hat{w}} .
\end{aligned}
$$

Similar to (2.49), we have

$$
\begin{aligned}
& \int_{\Omega}\left\langle\left[A_{1}-A_{2}\right] \nabla v_{2, \delta}, \nabla \hat{w}\right\rangle+\left\langle\left[A_{1}-A_{2}\right] \nabla v_{2, \delta}, \nabla \hat{v}_{2}\right\rangle+\lambda_{0}\left(\Sigma_{1}-\Sigma_{2}\right) v_{2, \delta} \overline{\hat{v}}_{2} \\
& \quad=\int_{\Omega} g_{2} \overline{\hat{w}}-\left(\overline{\hat{g}}_{1}-\overline{\hat{g}}_{2}\right) v_{2, \delta}+\overline{\hat{G}}_{1} \nabla v_{2}+\lambda_{0}\left(\Sigma_{2}-\Sigma_{1}\right) v_{2, \delta} \overline{\hat{w}} .
\end{aligned}
$$

There is no term $(1+\delta)$ in front of $A_{1}$ and $\Sigma_{1}$ in (4.36) since we only use the equation of $v_{2}$ and the system of $\hat{v}$ here. Choosing $\delta=\delta_{n}$, letting $n \rightarrow+\infty$ in (4.35) and (4.36) and using (4.34), we obtain (4.31) and (4.32). Therefore, $v$ is a viscosity solution.

The uniqueness of viscosity solutions is now standard from its definition as in the proof of Proposition 5.

Set

$$
\hat{\mathcal{H}}_{0}(\Omega):=\left\{\left(v_{1}, v_{2}\right) \in\left[L_{l o c}^{2}(\Omega)\right]^{2} ;\left\|\left(v_{1}, v_{2}\right)\right\|_{\hat{\mathcal{H}}_{0}(\Omega)}<+\infty\right\},
$$

where

$$
\langle u, v\rangle_{\hat{\mathcal{H}}_{0}(\Omega)}=\int_{\Omega \backslash \Omega_{\tau}} \nabla u_{2} \nabla \bar{v}_{2}+\int_{\Omega} d_{\Gamma}^{-\beta_{1}}\left(u_{1}-u_{2}\right)\left(\bar{v}_{1}-\bar{v}_{2}\right)+\int_{\Omega} d_{\Gamma}^{\beta_{1}} u \bar{v} .
$$


Fix $\lambda_{0}>\Lambda_{0}$ where $\Lambda_{0}$ is the constant in Proposition 9. Define

$$
\begin{aligned}
T_{3, \lambda}: & \hat{\mathcal{H}}_{0}(\Omega) \rightarrow \hat{\mathcal{H}}_{0}(\Omega) \\
\left(f_{1}, f_{2}\right) & \mapsto\left(u_{1}, u_{2}\right),
\end{aligned}
$$

where $\left(u_{1}, u_{2}\right) \in \hat{\mathcal{H}}_{1}(\Omega)$ is the unique viscosity solution of (4.8) with

$$
g_{1}=\Sigma_{1}\left(f_{1}-f_{2}\right)+\Sigma_{2} f_{2}, \quad G_{1}=\lambda^{-1}\left(A_{1}-A_{2}\right) \nabla f_{2}, \quad \text { and } \quad g_{2}=\Sigma_{2} f_{2} .
$$

We have

Lemma 16 There exists $\Lambda_{0}>1$ such that if $\lambda_{0}>\Lambda_{0}$ then $T_{3, \lambda}$ is a compact operator.

Proof Let $\left(f_{1, n}, f_{2, n}\right)$ be an arbitrary bounded sequence in $\hat{\mathcal{H}}_{0}(\Omega)$ and set

$$
\left(u_{1, n}, u_{2, n}\right)=T_{3, \lambda}\left(f_{1, n}, f_{2, n}\right) .
$$

Denote $g_{1, n}, g_{2, n}, G_{1, n}$ as in (4.40) where $\left(f_{1}, f_{2}\right)$ is replaced by $\left(f_{1, n}, f_{2, n}\right)$. It follows from (4.33) that $\left(u_{1, n}, u_{2, n}\right)$ is bounded sequence in $\hat{\mathcal{H}}_{1}(\Omega)$. Using the equations of $u_{1, n}$ and $u_{2, n}$, we derive that $\left(u_{1, n}, u_{2, n}\right)$ is bounded in $H_{l o c}^{1}(\Omega)$. Since $\operatorname{div}\left(A_{2} \nabla u_{2, n}\right)-\lambda_{0} \Sigma_{2} u_{2, n}=$ $g_{2, n},\left(u_{2, n}\right)$ is bounded in $H_{l o c}^{1}(\Omega)$, and $\left(g_{2, n}\right)$ is bounded in $L_{l o c}^{2}(\Omega)$, one might assume that

$$
\left(u_{2, n}\right) \text { converges strongly in } H_{l o c}^{1}(\Omega) \text {. }
$$

By Hardy's inequality, we have, with $w_{n}=u_{1, n}-u_{2, n}$,

$$
\int_{\Omega} d_{\Gamma}^{-2}\left|w_{n}\right|^{2} \leq C_{\Omega} \int_{\Omega}\left|\nabla w_{n}\right|^{2} .
$$

Since the embedding $H^{1} \subset L^{2}$ is compact, applying Lemma 17 below to $\psi_{n}=w_{n}, s=-2$, and $t=-\beta_{1}$, one might assume in addition that

$$
\left(w_{n}\right) \text { converges in } L^{2}\left(\Omega, d_{\Gamma}^{-\beta_{1}}\right) .
$$

Since the embedding $H^{1} \subset L^{2}$ is compact, applying Lemma 17 below to $\psi_{n}=u_{n}, s=\beta$, and $t=\beta_{1}>\beta$, one might assume in addition that

$$
\left(u_{n}\right) \text { converges in }\left[L^{2}\left(\Omega, d_{\Gamma}^{\beta_{1}}\right)\right]^{2} .
$$

By Lemma 6, we have

$$
\int_{\Omega} d_{\Gamma}^{\beta+2}\left|\nabla u_{2, n}\right|^{2} \leq C \int_{\Omega} d_{\Gamma}^{\beta}\left|u_{2, n}\right|^{2}+d_{\Gamma}^{\beta}\left|g_{2, n}\right|^{2} \leq C .
$$

Using (4.41) and applying Lemma 17 to $\psi_{n}=\nabla u_{2, n}, s=\beta+2$, and $t=\beta_{1}+2$, one can assume in addition that

$$
\nabla u_{2, n} \text { converges in }\left[L^{2}\left(\Omega, d_{\Gamma}^{\beta_{1}+2}\right)\right]^{d} .
$$

The convergence of $\left(u_{n}\right)$ in $\hat{\mathcal{H}}_{0}(\Omega)$ now follows from (4.41), (4.42), (4.43), and (4.44).

Remark 8 The embedding $\hat{\mathcal{H}}_{1}(\Omega)$ into $\hat{\mathcal{H}}_{0}(\Omega)$ is not compact. Nevertheless, $T_{3, \lambda}$ is compact as shown in Lemma 16.

In the proof of Lemma 16, we used the following compactness result. 
Lemma 17 Let $m \geq 1$ and $\left(\psi_{n}\right) \subset\left[L_{\text {loc }}^{2}(\Omega)\right]^{m}$ be such that for every $K \subset \subset \Omega,\left(\left.\psi_{n}\right|_{K}\right)$ is relatively compact in $\left[L^{2}(K)\right]^{m}$. Assume that, for some $s \in \mathbb{R}$,

$$
\sup _{n}\left\|\psi_{n}\right\|_{\left[L^{2}\left(\Omega, d_{\Gamma}^{s}\right)\right]^{m}}<+\infty .
$$

Then $\left(\psi_{n}\right)$ is relatively compact in $\left[L^{2}\left(\Omega, d_{\Gamma}^{t}\right)\right]^{m}$ for every $t>s$.

Proof The proof is almost in the spirit of the one [23, Lemma 7]. For the sake of completeness, we present the proof. Set

$$
C=\sup _{n}\left\|\psi_{n}\right\|_{\left[L^{2}\left(\Omega, d_{\Gamma}^{s}\right)\right]^{m}}^{2} .
$$

We have, for $\tau>0$,

$$
\int_{\Omega_{\tau}} d_{\Gamma}^{t}\left|\psi_{n}\right|^{2} \leq \tau^{t-s} \int_{\Omega_{\tau}} d_{\Gamma}^{s}\left|\psi_{n}\right|^{2} \leq C \tau^{t-s} .
$$

Fix $\varepsilon>0$ arbitrary. Fix $\tau>0$ small enough such that

$$
\left\|\psi_{n}\right\|_{L^{2}\left(\Omega_{\tau}, d_{\Gamma}^{t}\right)} \leq \varepsilon / 2 \quad \forall n \in \mathbb{N} .
$$

Such a constant $\tau$ exists by (4.45). Since $\left(\psi_{n}\right)$ is relatively compact in $L^{2}\left(\Omega \backslash \Omega_{\tau}\right)$, there exist $\psi_{n_{1}}, \cdots, \psi_{n_{k}}$ such that

$$
\left\{\psi_{n} \in L^{2}\left(\Omega \backslash \Omega_{\tau}\right) ; n \in \mathbb{N}\right\} \subset \bigcup_{j=1}^{k}\left\{\psi \in L^{2}\left(\Omega \backslash \Omega_{\tau}\right) ;\left\|\psi-\psi_{n_{j}}\right\|_{L^{2}\left(\Omega \backslash \Omega_{\tau}, d_{\Gamma}^{t}\right)} \leq \varepsilon / 2\right\} .
$$

A combination of (4.46) and (4.47) yields

$$
\left\{\psi_{n} \in L^{2}\left(\Omega, d_{\Gamma}^{t}\right) ; n \in \mathbb{N}\right\} \subset \bigcup_{j=1}^{k}\left\{\psi \in L^{2}(\Omega) ;\left\|\psi-\psi_{n_{j}}\right\|_{L^{2}\left(\Omega, d_{\Gamma}^{t}\right)} \leq 3 \varepsilon / 2\right\} .
$$

Therefore, $\left(\psi_{n}\right)$ is relatively compact in $L^{2}\left(\Omega, d_{\Gamma}^{t}\right)$.

We are ready to give

Proof of Theorem 3 By the spectral theory of a family of compact analytic operators, the spectrum of $T_{3, \lambda}$ is discrete (see, e.g., [27, Theorem 8.92]). The conclusion follows by the definition of $T_{3, \lambda}$.

\section{On the case $A_{1}$ and $A_{2}$ satisfying the complementary condition on $\Gamma$ : Proof of Theorem 4}

This section is devoted to the proof of Theorem 4. We first establish the well-posedness in $\left[H^{1}(\Omega)\right]^{2}$ of the following system (Proposition 10), for $\lambda_{0}>0$ (large),

$$
\begin{cases}\operatorname{div}\left(A_{1} \nabla u_{1}\right)-i \lambda_{0} \Sigma_{1} u_{1}=g_{1} & \text { in } \Omega, \\ \operatorname{div}\left(A_{2} \nabla u_{2}\right)-i \lambda_{0} \Sigma_{2} u_{2}=g_{2} & \text { in } \Omega \\ u_{1}=u_{2}, \quad A_{1} \nabla u_{1} \cdot v=A_{2} \nabla u_{2} \cdot v & \text { on } \Gamma\end{cases}
$$

for a given pair $\left(g_{1}, g_{2}\right) \in\left[L^{2}(\Omega)\right]^{2}$ under the following three assumptions, which are the assumptions of Theorem 4: 
C1) $A_{1}, A_{2}, \Sigma_{1}, \Sigma_{2}$ are continuous in a neighbourhood of $\Gamma$.

C2) For all $x \in \Gamma, A_{1}(x)$ and $A_{2}(x)$ satisfy the following condition, with $e=v(x)$,

$\left\langle A_{2}(x) e, e\right\rangle\left\langle A_{2}(x) \xi, \xi\right\rangle-\left\langle A_{2}(x) e, \xi\right\rangle^{2} \neq\left\langle A_{1}(x) e, e\right\rangle\left\langle A_{1}(x) \xi, \xi\right\rangle-\left\langle A_{1}(x) e, \xi\right\rangle^{2}$ for all $\xi \in \mathcal{P}(x) \backslash\{0\}$, where

$$
\mathcal{P}(x):=\left\{\xi \in \mathbb{R}^{d} ;\langle\xi, e\rangle=0\right\} .
$$

C3) For all $x \in \Gamma, A_{1}(x), A_{2}(x), \Sigma_{1}(x)$, and $\Sigma_{2}(x)$ verify

$$
\left\langle A_{1}(x) v(x), v(x)\right| \Sigma_{1}(x) \neq\left\langle A_{2}(x) v(x), v(x)\right| \Sigma_{2}(x) .
$$

We then define the operator

$$
\begin{aligned}
T_{4}\left(f_{1}, f_{2}\right) & =\left(u_{1}, u_{2}\right) \text { where }\left(u_{1}, u_{2}\right) \text { is the unique solution of }(5.1) \text { with }\left(g_{1}, g_{2}\right) \\
& =\left(\Sigma_{1} f_{1}, \Sigma_{2} f_{2}\right) .
\end{aligned}
$$

The basic ingredient in the proof of Proposition 10 is the following lemma:

Lemma 18 Let $\lambda \geq 1, A_{1}, A_{2}$ be two constant positive symmetric matrices and let $\Sigma_{1}, \Sigma_{2}$ be two positive constants. Assume that

$$
\left\langle A_{2} e_{d}, e_{d}\right\rangle\left\langle A_{2} \xi, \xi\right\rangle-\left\langle A_{2} e_{d}, \xi\right\rangle^{2} \neq\left\langle A_{1} e_{d}, e_{d}\right\rangle\left\langle A_{1} \xi, \xi\right\rangle-\left\langle A_{1} e_{d}, \xi\right\rangle^{2}
$$

for all $\xi \in P \backslash\{0\}$, where

$$
\mathcal{P}:=\left\{\xi \in \mathbb{R}^{d} ;\left\langle\xi, e_{d}\right\rangle=0\right\}
$$

and

$$
\left\langle A_{1} e_{d}, e_{d}\right| \Sigma_{1} \neq\left\langle A_{2} e_{d}, e_{d}\right\rangle \Sigma_{2}
$$

Let $g=\left(g_{1}, g_{2}\right) \in\left[L^{2}\left(\mathbb{R}_{+}^{d}\right)\right]^{2}, G=\left(G_{1}, G_{2}\right) \in\left(\left[L^{2}\left(\mathbb{R}_{+}^{d}\right)\right]^{d}\right)^{2}, \varphi \in H^{1 / 2}\left(\mathbb{R}_{0}^{d}\right), \phi \in$ $H^{-1 / 2}\left(\mathbb{R}_{0}^{d}\right)$. Assume that $v=\left(v_{1}, v_{2}\right) \in\left[H^{1}\left(\mathbb{R}_{+}^{d}\right)\right]^{2}$ is a solution of the system

$$
\begin{cases}\operatorname{div}\left(A_{1} \nabla v_{1}\right)-i \lambda \Sigma_{1} v_{1}=g_{1}+\operatorname{div}\left(G_{1}\right) & \text { in } \mathbb{R}_{+}^{d}, \\ \operatorname{div}\left(A_{2} \nabla v_{2}\right)-i \lambda \Sigma_{2} v_{2}=g_{2}+\operatorname{div}\left(G_{2}\right) & \text { in } \mathbb{R}_{+}^{d}, \\ v_{1}-v_{2}=\varphi,\left(A_{1} \nabla v_{1}-G_{1}\right) \cdot e_{d}-\left(A_{2} \nabla v_{2}-G_{2}\right) \cdot e_{d}=\phi & \text { on } \mathbb{R}_{0}^{d}\end{cases}
$$

We have

$$
\begin{aligned}
& \|v\|_{H^{1}\left(\mathbb{R}_{+}^{d}\right)}+\lambda^{1 / 2}\|v\|_{L^{2}\left(\mathbb{R}_{+}^{d}\right)} \\
& \quad \leq C\left(\lambda^{-1 / 2}\|g\|_{L^{2}\left(\mathbb{R}_{+}^{d}\right)}+\|G\|_{L^{2}\left(\mathbb{R}_{+}^{d}\right)}+\|\varphi\|_{H^{1 / 2}\left(\mathbb{R}_{0}^{d}\right)}+\lambda^{1 / 4}\|\varphi\|_{L^{2}\left(\mathbb{R}_{0}^{d}\right)}+\|\phi\|_{H^{-1 / 2}\left(\mathbb{R}_{0}^{d}\right)}\right),
\end{aligned}
$$

where $C$ is a positive constant depending only on $d, A_{1}, A_{2}, \Sigma_{1}$, and $\Sigma_{2}$.

Here and in what follows in this section,

$$
\begin{aligned}
& \mathbb{R}_{+}^{d}:=\left\{x=\left(x^{\prime}, x_{d}\right) \in \mathbb{R}^{d-1} \times \mathbb{R} ; x_{d}>0\right\} \quad \text { and } \\
& \mathbb{R}_{0}^{d}:=\left\{x=\left(x^{\prime}, x_{d}\right) \in \mathbb{R}^{d-1} \times \mathbb{R} ; x_{d}=0\right\} .
\end{aligned}
$$

Proof Let $u_{j} \in H^{1}\left(\mathbb{R}_{+}^{d}\right)(j=1,2)$ be the unique solution of the system

$$
\operatorname{div}\left(A_{j} \nabla u_{j}\right)-i \lambda \Sigma_{j} u_{j}=g_{j}+\operatorname{div}\left(G_{j}\right) \quad \text { in } \mathbb{R}_{+}^{d}, \quad \text { and } \quad\left(A_{j} \nabla u_{j}-G_{j}\right) \cdot e_{d}=\phi_{j},
$$


with $\phi_{1}=\phi$ and $\phi_{2}=0$. Multiplying the equation of $u_{j}$ by $\bar{u}_{j}$ and integrating on $\mathbb{R}_{+}^{d}$, we have

$$
\int_{\mathbb{R}_{+}^{d}}\left\langle A_{j} \nabla u_{j}, \nabla u_{j}\right\rangle+i \lambda \Sigma_{j}\left|u_{j}\right|^{2}=\int_{\mathbb{R}_{+}^{d}} G_{j} \nabla \bar{u}_{j}-g_{j} \bar{u}_{j}+\int_{\mathbb{R}_{0}^{d}} \phi_{j} \bar{u}_{j} ;
$$

which implies

$$
\left\|\nabla u_{j}\right\|_{L^{2}\left(\mathbb{R}_{+}^{d}\right)}+\lambda^{1 / 2}\left\|u_{j}\right\|_{L^{2}\left(\mathbb{R}_{+}^{d}\right)} \leq C\left(\lambda^{-1 / 2}\left\|g_{j}\right\|_{L^{2}\left(\mathbb{R}_{+}^{d}\right)}+\left\|G_{j}\right\|_{L^{2}\left(\mathbb{R}_{+}^{d}\right)}+\left\|\phi_{j}\right\|_{H^{-1 / 2}\left(\mathbb{R}_{0}^{d}\right)}\right) .
$$

Since, for $j=1,2$,

$$
\int_{\mathbb{R}^{d-1}}\left|u_{j}\left(x^{\prime}\right)\right|^{2} d x^{\prime} \leq 2 \int_{\mathbb{R}_{+}^{d}}\left|\partial_{x_{d}} u_{j}\right|\left|u_{j}\right|,
$$

it follows that, for $j=1,2$,

$$
\left\|u_{j}\right\|_{L^{2}\left(\mathbb{R}_{0}^{d}\right)}^{2} \leq 2\left\|u_{j}\right\|_{L^{2}\left(\mathbb{R}_{+}^{d}\right)}\left\|\nabla u_{j}\right\|_{L^{2}\left(\mathbb{R}_{+}^{d}\right)} .
$$

A combination of (5.8) and (5.9) yields, for $j=1,2$,

$$
\begin{aligned}
& \left\|u_{j}\right\|_{H^{1}\left(\mathbb{R}_{+}^{d}\right)}+\lambda^{1 / 2}\left\|u_{j}\right\|_{L^{2}\left(\mathbb{R}_{+}^{d}\right)}+\left\|u_{j}\right\|_{H^{1 / 2}\left(\mathbb{R}_{0}^{d}\right)}+\lambda^{1 / 4}\left\|u_{j}\right\|_{L^{2}\left(\mathbb{R}_{0}^{d}\right)} \\
& \leq C\left(\lambda^{-1 / 2}\left\|g_{j}\right\|_{L^{2}\left(\mathbb{R}_{+}^{d}\right)}+\left\|G_{j}\right\|_{L^{2}\left(\mathbb{R}_{+}^{d}\right)}+\left\|\phi_{j}\right\|_{H^{-1 / 2}}\right) .
\end{aligned}
$$

By considering the system of $\left(v_{1}-u_{1}, v_{2}-u_{2}\right)$, one might assume that $g_{1}=g_{2}=0, G_{1}=$ $G_{2}=0$, and $\phi=0$. In what follows, we make this assumption.

Let $\hat{v}_{j}\left(\xi^{\prime}, t\right)$ for $j=1,2$ and $\hat{\varphi}\left(\xi^{\prime}, t\right)$ be the Fourier transform of $v_{j}$ and $\varphi$ with respect to $x^{\prime} \in \mathbb{R}^{d-1}$, i.e., for $\left(\xi^{\prime}, t\right) \in \mathbb{R}^{d-1} \times(0,+\infty)$,

$$
\begin{aligned}
\hat{v}_{j}\left(\xi^{\prime}, t\right) & =\int_{\mathbb{R}^{d-1}} v_{j}\left(x^{\prime}, t\right) e^{-i x^{\prime} \cdot \xi^{\prime}} d x^{\prime} \quad \text { for } j=1,2, \quad \text { and } \\
\hat{\varphi}\left(\xi^{\prime}, t\right) & =\int_{\mathbb{R}^{d-1}} \varphi\left(x^{\prime}\right) e^{-i x^{\prime} \cdot \xi^{\prime}} d x^{\prime} .
\end{aligned}
$$

Since

$$
\operatorname{div}\left(A_{j} \nabla v_{j}\right)-i \lambda \Sigma_{j} v_{j}=0 \text { in } \mathbb{R}_{+}^{d},
$$

it follows that

$$
a_{j} \hat{v}_{j}^{\prime \prime}(t)+2 i b_{j} \hat{v}_{j}^{\prime}(t)-\left(c_{j}+i \lambda \Sigma_{j}\right) \hat{v}_{j}(t)=0 \text { for } t>0
$$

where

$$
a_{j}=\left(A_{j}\right)_{d, d}, \quad b_{j}=\sum_{k=1}^{d-1}\left(A_{j}\right)_{d, k} \xi_{k}, \quad \text { and } \quad c_{j}=\sum_{k=1}^{d-1} \sum_{l=1}^{d-1}\left(A_{j}\right)_{k, l} \xi_{k} \xi_{l} .
$$

Here $\left(A_{j}\right)_{k, l}$ denotes the $(k, l)$ component of $A_{j}$ for $j=1,2$ and the symmetry of $A_{j}$ is used. Define, for $j=1,2$,

$$
\Delta_{j}=-b_{j}^{2}+a_{j}\left(c_{j}+i \lambda \Sigma_{j}\right)
$$

Denote $\xi=\left(\xi^{\prime}, 0\right)$. Since $A_{j}$ is symmetric and positive, it is clear that, for $j=1,2$,

$$
\begin{aligned}
a_{j} & =\left\langle A_{j} e_{d}, e_{d}\right\rangle>0, \quad b_{j}=\left\langle A_{j} \xi, e_{d}\right\rangle, \quad \text { and } \\
a_{j} c_{j}-b_{j}^{2} & =\left\langle A_{j} e_{d}, e_{d}\right\rangle\left\langle A_{j} \xi, \xi\right\rangle-\left\langle A_{j} e_{d}, \xi\right\rangle^{2}>0 .
\end{aligned}
$$


For $j=1,2$, let $\sqrt{\Delta_{j}}$ denote the square root of $\Delta_{j}$ with positive real part and set

$$
\eta_{j}=\left(-i b_{j}-\sqrt{\Delta_{j}}\right) / a_{j}
$$

Since $\hat{v}_{j}\left(\xi^{\prime}, t\right) \in L^{2}\left(\mathbb{R}_{+}^{d}\right)$, we derive from (5.11) that, for $j=1,2$,

$$
\hat{v}_{j}\left(\xi^{\prime}, t\right)=\alpha_{j}\left(\xi^{\prime}\right) e^{\eta_{j} t},
$$

for some $\alpha_{j} \in L^{2}\left(\mathbb{R}_{0}^{d}\right)$. Using the fact that $v_{1}-v_{2}=\varphi$ and $A_{1} \nabla v_{1} \cdot e_{d}-A_{2} \nabla v_{2} \cdot e_{d}=0$ on $\mathbb{R}_{0}^{d}$, we derive that

$\alpha_{1}\left(\xi^{\prime}\right)-\alpha_{2}\left(\xi^{\prime}\right)=\hat{\varphi}\left(\xi^{\prime}\right) \quad$ and $\quad \alpha_{1}\left(\xi^{\prime}\right)\left\langle i A_{1} \xi+\eta_{1} A_{1} e_{d}, e_{d}\right\rangle-\alpha_{2}\left(\xi^{\prime}\right)\left\langle i A_{2} \xi+\eta_{2} A_{2} e_{d}, e_{d}\right\rangle=0$.

Since, by (5.14),

$$
\left\langle A_{j} \xi, e_{d}\right\rangle-\left\langle A_{j} e_{d}, e_{d}\right\rangle b_{j} / a_{j}=0 \quad \text { for } j=1,2,
$$

the last identity of (5.16) implies

$$
\alpha_{1}\left(\xi^{\prime}\right) \sqrt{\Delta_{1}}=\alpha_{2}\left(\xi^{\prime}\right) \sqrt{\Delta_{2}} .
$$

Combining this identity and the first one of (5.16) yields

$$
\alpha_{1}\left(\xi^{\prime}\right)=\frac{\hat{\varphi}\left(\xi^{\prime}\right) \sqrt{\Delta_{2}}}{\sqrt{\Delta_{2}}-\sqrt{\Delta_{1}}} .
$$

Note that, by (5.4), (5.5), (5.13), and (5.14),

$$
\begin{aligned}
\left|\Delta_{2}-\Delta_{1}\right|^{2}= & \left(\left\langle A_{2} e_{d}, e_{d}\right\rangle\left\langle A_{2} \xi, \xi\right\rangle-\left\langle A_{2} e_{d}, \xi\right\rangle^{2}-\left\langle A_{1} e_{d}, e_{d}\right\rangle\left\langle A_{1} \xi, \xi\right\rangle+\left\langle A_{1} e_{d}, \xi\right\rangle^{2}\right)^{2} \\
& +\lambda^{2}\left(\left\langle A_{1} e_{d}, e_{d}\right| \Sigma_{1}-\left\langle A_{2} e_{d}, e_{d}\right| \Sigma_{2}\right)^{2} \\
\geq & C\left(|\xi|^{4}+\lambda^{2}\right)
\end{aligned}
$$

and

$$
\left|\Delta_{j}\right| \leq C\left(|\xi|^{2}+\lambda\right)
$$

This implies

$$
\frac{\sqrt{\Delta_{2}}}{\left|\sqrt{\Delta_{2}}-\sqrt{\Delta_{1}}\right|}=\frac{\sqrt{\Delta_{2}}\left(\sqrt{\Delta_{2}}+\sqrt{\Delta_{1}}\right)}{\left|\Delta_{2}-\Delta_{1}\right|} \leq C .
$$

Since, by Parseval's theorem,

$$
\int_{\mathbb{R}_{+}^{d}}\left|v_{1}\right|^{2}=C_{d} \int_{0}^{\infty} \int_{\mathbb{R}^{d-1}}\left|\hat{v}_{1}\left(\xi^{\prime}, t\right)\right|^{2} d \xi^{\prime} d t,
$$

it follows from (5.15), (5.17), and (5.18) that

$$
\int_{\mathbb{R}_{+}^{d}}\left|v_{1}\right|^{2} \leq C \int_{\mathbb{R}^{d-1}} \int_{0}^{\infty}\left|\hat{\varphi}\left(\xi^{\prime}\right)\right|^{2}\left|e^{2 \eta_{1} t}\right| d t d \xi^{\prime} .
$$

Using the fact

$$
\int_{0}^{\infty}\left|e^{2 \eta_{1} t}\right| d t \leq \frac{1}{2\left|\Re\left(\eta_{1}\right)\right|} \quad \text { and } \quad \frac{1}{\left|\Re\left(\eta_{1}\right)\right|} \leq \frac{C}{\left|\xi^{\prime}\right|+\sqrt{\lambda}}
$$


we deduce from (5.19) that

$$
\int_{\mathbb{R}_{+}^{d}}\left|v_{1}\right|^{2} \leq \int_{\mathbb{R}^{d-1}} \frac{C\left|\hat{\varphi}\left(\xi^{\prime}\right)\right|^{2}}{\left|\xi^{\prime}\right|+\sqrt{\lambda}} d \xi^{\prime} \leq C \lambda^{-1 / 2}\|\varphi\|_{L^{2}\left(\mathbb{R}_{0}^{d}\right)}^{2} .
$$

which yields the corresponding estimate for $\left\|v_{1}\right\|_{L^{2}\left(\mathbb{R}_{+}^{d}\right)}$. The estimates follows similarly. The details are left to the reader.

Remark 9 The computation of $\hat{v}_{j}$ in Lemma 18 has roots from the proof of [23, Proposition 1].

Proposition 10 Assume (1), C2), and C3). There exists a constant $\Lambda_{0}>0$ depending only on $A_{1}, A_{2}, \Sigma_{1}, \Sigma_{2}$, and $\Omega$, such that for $\lambda>\Lambda_{0}$ and $g=\left(g_{1}, g_{2}\right) \in\left[L^{2}(\Omega)\right]^{2}$, there exists a unique solution $v=\left(v_{1}, v_{2}\right) \in\left[H^{1}(\Omega)\right]^{2}$ satisfy

$$
\begin{cases}\operatorname{div}\left(A_{1} \nabla v_{1}\right)-i \lambda \Sigma_{1} v_{1}=g_{1} & \text { in } \Omega, \\ \operatorname{div}\left(A_{2} \nabla v_{2}\right)-i \lambda \Sigma_{2} v_{2}=g_{2} & \text { in } \Omega, \\ v_{1}-v_{2}=0, \quad A_{1} \nabla v_{1} \cdot v-A_{2} \nabla v_{2} \cdot v=0 & \text { on } \Gamma .\end{cases}
$$

Moreover,

$$
\|v\|_{H^{1}(\Omega)}+\lambda^{1 / 2}\|v\|_{L^{2}(\Omega)} \leq C \lambda^{-1 / 2}\|g\|_{L^{2}(\Omega)},
$$

for some positive constant $C$ independent of $g$ and $\lambda$.

Proof We first assume the existence of $v \in\left[H^{1}(\Omega)\right]^{2}$ and derive the estimate for $v$. Assume that $\left(A_{1}, A_{2}, \Sigma_{1}, \Sigma_{2}\right) \in C\left(\bar{\Omega}_{\tau_{0}}\right)$ for some $\tau_{0}>0$. Using local charts, applying Lemma 18 with $\varphi=0$ and $\phi=0$, and involving the standard freezing coefficient technique, we have

$$
\|v\|_{H^{1}\left(\Omega_{\tau}\right)}+\lambda^{1 / 2}\|v\|_{L^{2}\left(\Omega_{\tau}\right)} \leq C\left(\|v\|_{H^{1}\left(\Omega_{2 \tau} \backslash \Omega_{\tau}\right)}+\lambda^{-1 / 2}\left\|\left(g_{1}, g_{2}\right)\right\|_{L^{2}(\Omega)}\right),
$$

if $\tau>0$ is small enough and $\lambda>1$. Here and in what follows $C$ denotes a positive constant independent of $v, g$, and $\lambda$; $C$ depends on $\left(A_{1}, A_{2}, \Sigma_{1}, \Sigma_{2}\right), \Omega$, and $\tau$. Fix such a positive constant $\tau$. Applying Lemma 9 to $v$, we have

$$
\|v\|_{H^{1}\left(\Omega \backslash \Omega_{\tau}\right)} \leq c_{1} \exp \left(-c_{2} \sqrt{\lambda}\right)\|v\|_{L^{2}\left(\Omega_{\tau}\right)}+c_{1}\|g\|_{L^{2}(\Omega)},
$$

for some positive $c_{1}$ and $c_{2}$ independent of $\lambda, v$, and $g$. Combining (5.22) and (5.23) yields, for large $\lambda$,

$$
\|v\|_{H^{1}(\Omega)}+\lambda^{1 / 2}\|v\|_{L^{2}(\Omega)} \leq C\|g\|_{L^{2}(\Omega)}
$$

which is (5.21). The existence of $v \in\left[H^{1}(\Omega)\right]^{2}$ follows from the uniqueness via the Fredholm theory by noting the well-posedness in $\left[H^{1}(\Omega)\right]^{2}$ of the system

$$
\begin{cases}\operatorname{div}\left(A_{1} \nabla v_{1}\right)-i \lambda \Sigma_{1} v_{1}=g_{1} & \text { in } \Omega, \\ \operatorname{div}\left(A_{2} \nabla v_{2}\right)+i \lambda \Sigma_{2} v_{2}=g_{2} & \text { in } \Omega, \\ v_{1}-v_{2}=0, \quad A_{1} \nabla v_{1} \cdot v-A_{2} \nabla v_{2} \cdot v=0 & \text { on } \Gamma,\end{cases}
$$

for $g \in\left[L^{2}(\Omega)\right]^{2}$ by Lax-Milgram's theory. The details are left to the reader.

We are ready to present

Proof of Theorem 4 Fix $\lambda>\Lambda_{0}$ where $\Lambda_{0}$ is the positive constant in Proposition 10. Define

$$
\begin{aligned}
T_{4}:\left[L^{2}(\Omega)\right]^{2} & \rightarrow\left[L^{2}(\Omega)\right]^{2} \\
\left(f_{1}, f_{2}\right) & \mapsto\left(u_{1}, u_{2}\right),
\end{aligned}
$$


where $\left(u_{1}, u_{2}\right) \in\left[H^{1}(\Omega)\right]^{2}$ is the unique solution of (5.1) with $\left(g_{1}, g_{2}\right)=\left(\Sigma_{1} f_{1}, \Sigma_{2} f_{2}\right)$. Since $H^{1}(\Omega) \subset L^{2}(\Omega)$ is compact, so $T_{4}$ is compact. The conclusion of Theorem 4 follows.

\section{References}

1. Agmon, S., Douglis, A., Nirenberg, L.: Estimates near the boundary for solutions of elliptic partial differential equations satisfying general boundary conditions. II. Commun. Pure Appl. Math. 17, 35-92 (1964)

2. Blåsten, E., Paivarinta, L.: Completeness of generalized transmission eigenstates. Inverse Probl. 29, 104002 (2013)

3. Bonnet-BenDhia, A.S., Chesnel, L., Haddar, H.: On the use of T-coercivity to study the interior transmission eigenvalue problem. C. R. Math. Acad. Sci. Paris 349, 647-651 (2011)

4. Brezis, H.: Functional Analysis, Sobolev Spaces and Partial Differential Equations. Universititex, Springer, Berlin (2010)

5. Cakoni, F., Colton, D., Haddar, H.: The linear sampling method for anisotropic media. J. Comput. Appl. Math. 146, 285-299 (2002)

6. Cakoni, F., Haddar, H.: Transmission eigenvalues in inverse scattering theory. Inside Out 60, 529-580 (2012)

7. Colton, D., Monk, P.: The inverse scattering problem for acoustic waves in an inhomogeneous medium. Quart. J. Mech. Appl. Math. 41, 97-125 (1988)

8. Colton, D., Kirsch, A., Paivarinta, L.: Far-field patterns for acoustic waves in an inhomogeneous medium. SIAM J. Math. Anal. 20, 1472-1483 (1989)

9. Colton, D., Paivarinta, L.: Transmission eigenvalues and a problem of Hans Lewy. J. Comput. Appl. Math. 117, 91-104 (2000)

10. Faierman, M.: Eigenvalue asymptotics for a boundary problem involving an elliptic system. Math. Nachr. 279, 1159-1184 (2006)

11. Haddar, H., Joly, P., Nguyen, H.-M.: Generalized impedance boundary conditions for scattering by strongly absorbing obstacles: the scalar case. Math. Models Methods Appl. Sci. 15, 1273-1300 (2005)

12. Hitrik, M., Krupchyk, K., Ola, P., Paivarinta, L.: The interior transmission problem and bounds on transmission eigenvalues. Math. Res. Lett. 18, 279-293 (2011)

13. Kirsch, A.: The denseness of the far field patterns for the transmission problem. IMA J. Appl. Math. 37, 213-225 (1986)

14. Lakshtanov, E., Vainberg, B.: Ellipticity in the interior transmission problem in anisotropic media. SIAM J. Math. Anal. 44, 1165-1174 (2012)

15. Lakshtanov, E., Vainberg, B.: Applications of elliptic opeator theory to the isotropic interior transmission eigenvalue problem. Inverse Probl. 29, 104003 (2013)

16. Robbiano, L.: Spectral analysis on interior transmission eigenvalues. Inverse Probl. 29, 104001 (2013)

17. Lopatinskii, Y.B.: On a method of reducing boundary problems for a system of differential equations of elliptic type to regular integral equations. Ukrain. Mat. Z 5, 123-151 (1953)

18. Nguyen, H.-M.: Asymptotic behavior of solutions to the Helmholtz equations with sign changing coefficients. Trans. Am. Math. Soc. 367, 6581-6595 (2015)

19. Nguyen, H.-M.: Superlensing using complementary media. Ann. Inst. H. Poincaré Anal. Non Linéaire 32, 471-484 (2015)

20. Nguyen, H.-M.: Cloaking via anomalous localized resonance. A connection between the localized resonance and the blow up of the power for doubly complementary media. C. R. Math. Acad. Sci. Paris 353, 41-46 (2015)

21. Nguyen, H.-M.: Cloaking via anomalous localized resonance for doubly complementary media in the quasi static regime. J. Eur. Math. Soc. 17, 1327-1365 (2015)

22. Nguyen, H-M.: Cloaking using complementary media in the quasistatic regime. Ann. Inst. H. Poincaré Anal. Non Linéaire. 33, 1509-1518 (2016)

23. Nguyen, H.-M.: Limiting absorption principle and well-posedness for the Helmholtz equation wiht sign changing coefficients. J. Math. Pures Appl. 106, 342-374 (2016)

24. Nguyen, H-M: Cloaking via anomalous localized resonance for doubly complementary media in the finite frequency regime. arXiv.org/abs/1511.08053

25. Nguyen, H-M.: Cloaking an arbitrary object via anomalous localized resonance: the cloak is independent of the object: the acoustic case. arXiv.1607.06492 
26. Nguyen, H.-M., Nguyen, H .L.: Cloaking using complementary media for the Helmholtz equation and a three spheres inequality for second order elliptic equations. Trans. Am. Math. Soc. Ser. B 2, 93-112 (2015)

27. Renardy, M., Rogers, R.C.: An introduction to partial differential equations. In: Texts in Applied Mathematics, vol. 13, 2nd ed. Springer, New York (2004)

28. Rynne, B.P., Sleeman, B.D.: The interior transmission problem and inverse scattering from inhomogeneous media. SIAM J. Math. Anal. 22, 1755-1762 (1991)

29. Sylvester, J.: Discreteness of transmission eigenvalues via upper triangular compact operators. SIAM J. Math. Anal. 44, 341-354 (2012) 